\title{
The "Virgo photometry catalogue"; a catalogue of 1180 galaxies in the direction of the Virgo Cluster's core
}

\author{
C.K. Young ${ }^{1,2}$ and M.J. Currie ${ }^{3}$ \\ ${ }^{1}$ Beijing Astronomical Observatory, Chinese Academy of Sciences, Beijing 100080, China \\ ${ }^{2}$ Department of Physics, University of Oxford, Nuclear and Astrophysics Laboratory, Keble Road, Oxford OX1 3RH, UK \\ ${ }^{3}$ Rutherford Appleton Laboratory, Chilton, Didcot, Oxfordshire OX11 0QX, UK
}

Received February 25; accepted May 22, 1997

\begin{abstract}
We present a new catalogue of galaxies in the direction of the Virgo Cluster's core: the Virgo Photometry Catalogue (VPC $)^{1}$. This catalogue contains 1180 galaxies (including background objects) within a 23 square-degree area of the sky centred on R.A.1950.0 $=$ $12^{\mathrm{h}} 26^{\mathrm{m}}$ and $\operatorname{dec} \cdot 1950.0=13^{\circ} 08^{\prime}$. The VPC galaxy sample comprises of non-stellar objects brighter than $B_{\mathrm{J}_{25}}=19.0$; the completeness limits being $B_{\mathrm{J}_{25}} \approx 18.5$ for the northern half of the survey area and $B_{\mathrm{J}_{25}} \approx 18.0$ for the southern half.

Independently-calibrated photographic surface photometry is presented for over 1000 galaxies in the $U, B_{\mathrm{J}}$ and $R_{\mathrm{C}}$ bands. Parameters listed for catalogued galaxies include: equatorial coordinates, morphological types, surface-brightness profile parameters (which preserve the majority of the original surface photometry information), $U, B_{\mathrm{J}} \& R_{\mathrm{C}}$ isophotal magnitudes, $B_{\mathrm{J}}$ and [transformed] $B$ total magnitudes, $\left(U-B_{\mathrm{J}}\right)$ and $\left(B_{\mathrm{J}}-R_{\mathrm{C}}\right)$ equal-area and total colours, apparent angular radii, ellipticities, position angles, heliocentric radial velocities and alternative designations. All total magnitudes and total colours are extrapolated according to a new system denoted $t$ in order to distinguish it from the $T$ system already in use.
\end{abstract}

The VPC is based primarily on four (one $U$, two $B_{\mathrm{J}}$ and one $R_{\mathrm{C}}$ ) UK-Schmidt plates, all of which were digitised using the Royal Observatory Edinburgh's (ROE) COSMOS measuring machine. All magnitudes, colours and surface-brightness parameters are derived from numerical integrations of segmented plate-scan data, except for (in 109 cases) saturated or (in 51 cases) inextricably-

Send offprint requests to: C.K. Young by e-mailing c.young1@physics.oxford.ac.uk

1 Appendices B, C and E, which contain the surface photometry, the main catalogue and the summary catalogue respectively, are only available in electronic form. They can be obtained from Le Centre de Données astronomiques de Strasbourg (CDS) via anonymous ftp to cdsarc.u-strasbg.fr (130.79.128.5) or via http://cdsweb.ustrasbg.fr/Abstract.html merged images; our segmentation software being able to cope with the vast majority of image mergers.

Key words: galaxies: clusters: individual: Virgo catalogues - galaxies: fundamental parameters galaxies: photometry

\section{Introduction}

The Virgo Cluster, being the dominant nearby cluster of galaxies, has long attracted much attention. It is irregular and exhibits significant substructure. Binggeli et al. (1987) divided Virgo into two main clusters: a dominant cluster, Cluster A, which is rich in early type galaxies and contains the giant elliptical NGC 4486 (otherwise known as M 87) and a secondary cluster, Cluster $B$, dominated by late spirals, in the direction of the giant elliptical NGC 4472 (M 49). Their Clusters A and B correspond to de Vaucouleurs's concentric $E \cup S$ and $S^{\prime}$ Clouds respectively (see e.g. de Vaucouleurs \& Corwin 1986). The VPC covers the central region of Cluster A/Clouds E\&S. It does not cover the central region of Cluster B/Cloud S', but there may of course still be many objects from this secondary cluster within the VPC survey area.

The equatorial coordinates of the centre of what is generally held to be the Virgo Cluster proper (i.e. of Cluster A or the E/S Clouds) are approximately $12^{\mathrm{h}} 26^{\mathrm{m}}$ in right ascension (1950.0) and $+13^{\circ} 00^{\prime}$ in declination (1950.0). The entire complex including substructures, is generally thought of as subtending a radius of about $6^{\circ}$ on the celestial sphere, though its true extent has yet to be established.

Radial velocities of the constituent galaxies vary from the most negative ones known (and the only negative ones known for galaxies outside the neighbourhood of the Local Group) to a maximum of about $+3000 \mathrm{~km} \mathrm{~s}^{-1}$, as is evident from Binggeli et al.'s (1985) Virgo Cluster Catalog 
(VCC). There appears to be a $\sim 4000 \mathrm{~km} \mathrm{~s}^{-1}$-deep velocity void behind the cluster, as the background system is at $+7000 \mathrm{~km} \mathrm{~s}^{-1}$ (see e.g. Drinkwater et al. 1996).

Most attempts to pin down the value of the Hubble constant have long been based on distance measurements for Virgo-Cluster galaxies. There are probably two main reasons for this. First, it is the nearest cluster in which a full complement of morphological types is represented: i.e. giant elliptical galaxies; classical ellipticals, spirals and irregulars; dwarf ellipticals and irregulars; blue compact dwarfs; galaxies in the process of merging and others that defy classification. It has therefore been possible to apply a wider variety of different distance indicators to Virgo galaxies than it has been for other clusters at similar redshifts such as Fornax or Leo. Second, Virgo has the added advantage of being easily observable from observatories in both the Northern and Southern hemispheres.

Virgo's popularity as a pivotal step in the cosmic distance scale has nevertheless not been without its difficulties and controversies. Until recently, the possibility that the spatial extent of the Virgo Cluster may have considerable line-of-sight depth was not taken seriously. Although Pierce \& Tully (1988) suggested the "possible presence of superposed foreground and background galaxies" in their sample of Virgo spirals, it was not until the distance-scale work of Tonry et al. (1990) and Tonry (1991) that any author was prepared to stand by the case for considerable depth. However, these authors did not confront the potentially embarrassing issue of the cluster's elongation ratio in the line of sight; which would have to exceed 5:1 in order to explain the depth in their derived galaxy distributions. Other authors have understandably tended to explain any apparent depth effect in Virgo galaxy distributions in terms of random observational errors and scatter intrinsic to the distance indicators employed.

The first authors not to avoid the elongation issue were Fukugita et al. (1993). These authors suggested that the Virgo Cluster's spiral-galaxy distribution is in fact filamentary in shape, rather than approximately spherically symmetric [as had been assumed before] with spirals lying at distances from $13 \mathrm{Mpc}$ all the way through to 30 Mpc. In Young \& Currie (1995) we also found a considerable depth effect, that we could not explain away in terms of errors or intrinsic scatter, this time in the distribution of dwarf-elliptical galaxies in the direction of the Virgo Cluster's core itself. Furthermore, we found preliminary evidence for a foreground concentration of galaxies several Mpc in front of the main cluster, which led us to suggest that the depth effect may be due to the projection of more than one distinct galaxy concentration onto the same sight line. This would avoid the need for elongation ratios of the order of $5: 1$.

This catalgoue has been compiled primarily with a view to investigating the Virgo Cluster's distance, threedimensional structure and kinematics; but will also provide the basis for a pencil beam survey in the Virgo direc- tion. We will also be investigating the luminosity functions of Virgo dwarfs, with the triple benefits of: galaxy samples selected according to objective criteria, independently calibrated machine generated magnitude measurements and the consideration of depth effects. These studies will be the subjects of future papers but a preliminary study of the spatial distribution of 64 Virgo dwarf-ellipticals has already been presented in Young \& Currie (1995) and a comparison with the existing magnitude scales for Virgo galaxies will be presented by Young et al. (in preparation).

\section{The observations}

Table 1. The UKST plates on which the VPC is based

\begin{tabular}{llll}
\hline plate & date & $(\alpha, \delta)_{1950.0}^{\mathrm{a}}$ & emulsion/filter/exposure \\
\hline U9362 & $84: 06: 03$ & $12: 27,13: 30$ & IIIaJ/UG1/3 hr. \\
J4882 & $79: 03: 23$ & $12: 25,13: 20$ & IIIaJ/GG395/1 hr. \\
J9229 & $84: 04: 26$ & $12: 27,13: 30$ & IIIaJ/GG395/1 hr. \\
R2936 & $77: 02: 27$ & $12: 25,13: 20$ & IIIaF/RG630/48 min. \\
\hline
\end{tabular}

${ }^{a}$ the equatorial coordinates of the plate centres: in hours and minutes of time for right ascension $(\alpha)$ and in degrees and minutes of arc for declination $(\delta)$.

The VPC is based on four photographic plates (one $U$, two $B_{\mathrm{J}}$ and one $R_{\mathrm{C}}$ ) of the same field; all of which were exposed on the 1.2-metre UK Schmidt Telescope (UKST) at Siding Spring Observatory in Australia. Details of all four plates are listed in Table 1. Each plate measured $356 \mathrm{~mm}$ square and covered an area of the sky 6.4 by 6.4 within an imaging area of dimensions $343 \mathrm{~mm}$ by $343 \mathrm{~mm}$. The plate scale was therefore $67.12 \mathrm{arcsec} / \mathrm{mm}$ in each case. All of the plates were hypersensitised by successive soaking in nitrogen and hydrogen gas. Those plates taken after 1982 December 3 had the added advantage of being nitrogen flushed; that is exposed under a constant stream of nitrogen. The significance of this flushing process is dealt with in Sects. 3.7 and 6.2.

\section{Data reduction procedure}

\subsection{The densitometry}

The plates were digitised by the ROE's Image and Data Processing Unit using its COSMOS measuring machine in mapping mode with a spot/pixel size of 32 microns. The central 4.8-square field of each plate was subdivided into four separate scanning fields to ensure that the resulting data files would be of a manageable size. The configurations of the scanning fields, which were labelled "A" through "D", are depicted in Fig. 1. Each field yielded a data array of dimensions 4096 by 4096 2.1arcsec-square pixels. As the position of each plate relative 


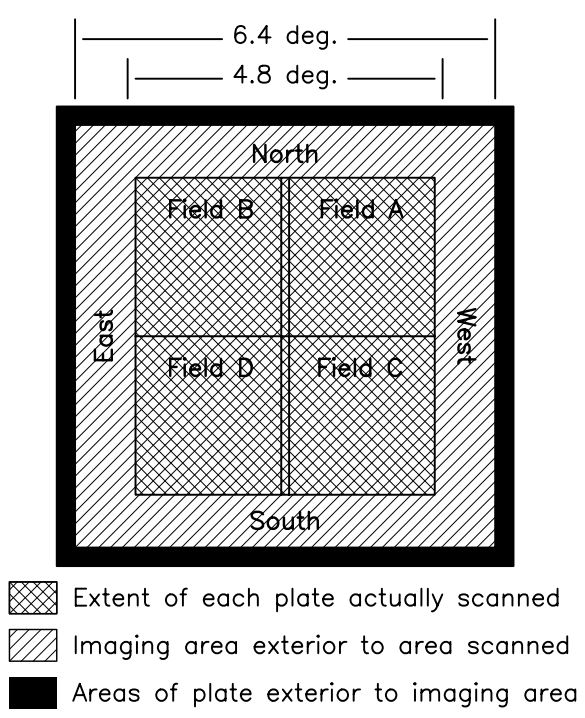

Fig. 1. Configuration of the square Virgo Fields A through D into which each plate was subdivided. Each field was the subject of a separate scan yielding a separate 4096 by 4096 pixel grid of transmission readings, though plates were not readjusted between scans of their different fields. Note that there was a 192-pixel-wide overlap between Fields A and B, and between $\mathrm{C}$ and D. Angular dimensions of the corresponding fields of view are indicated. Note that the positions of the peripheral areas containing the sensitometer spot sequences varied from plate to plate and have therefore not been shown, even though they were scanned

to the measuring-machine carriage remained unchanged throughout the scans of its four different fields, the $(x, y)$ coordinate systems of the different fields were not rotated with respect to one another; only the origins were shifted. Transformations between the coordinate systems of the same field on different plates, did however always require a degree of both shift and rotation as it was not possible to scan identical regions of different plates.

In addition to the main scans, the peripheral areas of the plates containing the sensitometer calibration spots were scanned too. As there were two sets of calibration spots per plate, two such scans were needed per plate.

The original scans of plates J4882 and R2936, that had been made in 1984, were found to be unsatisfactory (see Sect. 3.3) and re-scans were made in 1990 with a pixel size of 1 .'05 (though the resulting higher resolution data arrays were later binned up to the $2{ }^{\prime \prime} 1$ pixel size of the earlier scans). Several changes had been made to COSMOS during the intervening period such as increasing the cathoderay beam strength and upgrading the density-data format from 8 bit to 12 bit. These improvements also increased the machine's dynamic range.

\subsection{Relative calibration}

The initial relative-intensity calibrations were based on the sensitometer-spot scans. The UKST is presently fitted with two different step-wedge sensitometers; one sixteenstep device of Kitt Peak National Observatory (KPNO) design and one seven-step device. Originally the UKST carried two identical seven-step devices, but one of them was replaced by a KPNO sensitometer at the end of 1979. The step-wedge combinations and configurations of the plates relevant to this study are listed in Table 2. The seven-step wedges were illuminated by tungsten light bulbs whilst the KPNO projector uses a quartz-halogen lamp in conjunction with a BG34 colour-correction filter.

Table 2. The sensitometer combinations and configurations

\begin{tabular}{lll}
\hline plate & scan used & step wedges projected $^{\text {a }}$ \\
\hline U9362 & $1984 ;$ 8-bit & N edge:7-step, SE corner:16-step \\
J4882 & $1990 ; 12-$ bit & N edge:7-step, E edge:7-step \\
J9229 & $1984 ;$ 8-bit & N edge:7-step, SE corner:16-step \\
R2936 & $1990 ; 12-$ bit & N edge:7-step, E edge:7-step \\
\hline
\end{tabular}

a "N" for North, "SE" for South East and "E" for East.

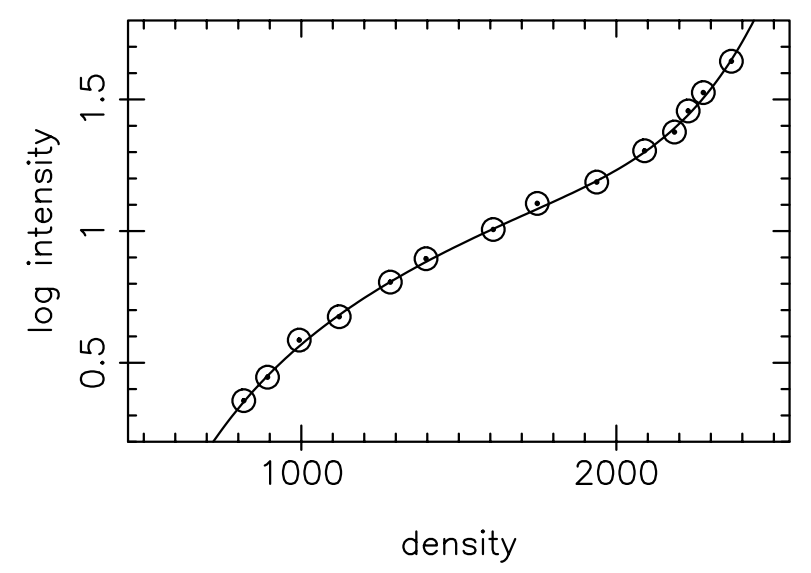

Fig. 2. The characteristic curve generated for plate R2936 by combining both of the plate's seven-spot sequences

A median emulsion-density value was obtained for each calibration spot from the digitised scan data. A characteristic curve was then generated for each spot sequence by fitting a polynomial function to plots of log spot density against log relative light intensity; values of the latter having been obtained directly from knowledge of the relative transmittances of the individual wedge components. The two characteristic curves for an individual plate could then be combined by sliding one in log relativeintensity space until the curves' residuals were minimised [in log relative-intensity space]. 3rd-degree or 5th-degree polynomial functions were then fitted to yield the final 
characteristic curves that were adopted. An example of one of the adopted curves is shown in Fig. 2.

In order to generate a two-dimensional polynomial surface function to model the mode sky density levels over each of the main scans, the central 4080-pixel square area of each 4096 pixel square grid was divided into an array of 48 by 48 square bins each containing 7225 pixels. A separate histogram of pixel-density values was constructed for each of the 2304 bins per scanning field. An optimum sky value was then obtained for each bin by kappa-sigma clipping (as first described by Godwin 1976 and Newell \& O'Neil 1977) of the data within each bin. After the initial rejection of pixel values exceeding $3 \times$ the typical sky density values (estimated roughly in the first instance by inspection of the digitised plate-scan data) the data for each bin were "clipped" four times, on each occasion discarding pixels deviating from the sample mean by more than a specified number of standard deviations: $1 \sigma$ on the first iteration, $2 \sigma$ on the second and third iterations and $2.5 \sigma$ on the fourth. The increasing severity of the rejection criteria was necessary because the sample standard deviations were inevitably reduced by each iteration. After the fourth iteration the new mean pixel value was adopted as the best estimate of the sky; on the grounds that it would correspond closely to the mode value provided that only a minority of the pixels within the bin were influenced by stellar or galaxy images. Obviously the latter assumption did not hold for those bins containing extended galaxy images and so further clipping of the resulting 48 by 48 array of sky estimates was necessary. The same kappa-sigma clipping algorithm with the same rejection criteria was subsequently applied to the 2304 sky estimates in order to preclude those bins containing the images of extended objects from influencing the final function used to model the sky. For each of the four scanning fields; a 4th degree [28-term] two-dimensional orthogonal polynomial function was fitted to the remaining estimated mode-density values (which corresponded typically to $93 \%$ of the bins). Individual pixel-density values were converted to relative intensities by means of the relevant characteristic curve and each was then divided by the local sky-value estimate (also in the same relative-intensity units) after this local sky-value estimate had first been subtracted. The subtraction was necessary in order to remove the night-sky contribution whilst the division served to counter "field effects" and "vignetting"; effects which are discussed in Sect. 7. The results were 4096-pixel-square arrays of intensity values in sky units (i.e. normalised to the sky brightness). It therefore remained to estimate the true sky brightness function for each plate in absolute units in order to provide a zero point for the relative-intensity scale.

\subsection{Absolute calibration}

Photoelectric aperture measurements, including simulated aperture CCD photometry of galaxies within the fields covered by the scan data, were used in order to determine the zero points of the relative-intensity scales. Ideally, all of the usable photoelectric measurements would have been compared directly with their photographic counterparts; the latter being obtained by the summation of pixelintensity values within circles of appropriate radius centred on the image centres. However, owing to saturation of the emulsion density within the cores of the majority of the galaxy images for which photoelectric photometry was available, and to limitations in the dynamic range of COSMOS, such a direct comparison was only possible in a handful of cases. In the vast majority of cases it was therefore necessary to compare intensities within annuli, and so provided the inner boundary of each annulus lay exterior to the saturated core region of the relevant image, the problem of core saturation could be overcome.

A total of $387 U$-, $658 \mathrm{~B}$-, $658 \mathrm{~V}$ - and $36 R_{\mathrm{C}}$-band published photoelectric-photometry measurements were collated for 65 galaxies within the field of interest from Longo \& de Vaucouleurs (1983) Longo \& de Vaucouleurs (1985) de Vaucouleurs \& Longo (1985) Burstein et al. (1987) and Poulain (1988). The $B$ and $V$ measurements were used to derive $B_{\mathrm{J}}$ values via the transformation:

$B_{\mathrm{J}}=0.65 B+0.35 \mathrm{~V}$

as used by e.g. Buta \& Corwin (1986).

A radial integrated-light profile was generated for each galaxy image for which there existed usable aperture photometry in the literature. As the scan data had already been reduced to a relative-intensity scale in sky units, these profiles could be computed in mag brighter than the sky. Each sum of an aperture measurement (in mag) and the modulus of the profile value (in mag above the sky) at the same radial distance, $\rho$, from the image centre therefore provided an estimate of the sky brightness at the position of the particular galaxy. The radial extent of each image's saturated region (if present) could therefore be estimated from plots of estimated sky brightness against $\rho$. Unreliable published aperture-photometry measurements could often be spotted at this stage: as data-points deviating significantly from the curve defined by the majority of the others. Outliers were only excluded however, if the other points had been obtained from several independent sources. Once the radius of the saturated core, $\rho_{\text {sat }}$, had been established for a plate image, all measurements made with apertures of less than $2 \rho_{\text {sat }}$ were culled from the lists of photometry allocated to that image.

Cored sky-surface-brightness estimates were then generated for each usable pair of published aperture measurements provided that the members of each pair were separated from one another in $\rho$ by at least 5 arcsec. The inner and outer radii of the annuli varied according to the availability of the published measurements. A mean sky brightness (in mag $\operatorname{arcsec}^{-2}$ ) and a standard deviation on the mean were then computed in intensity space for each 


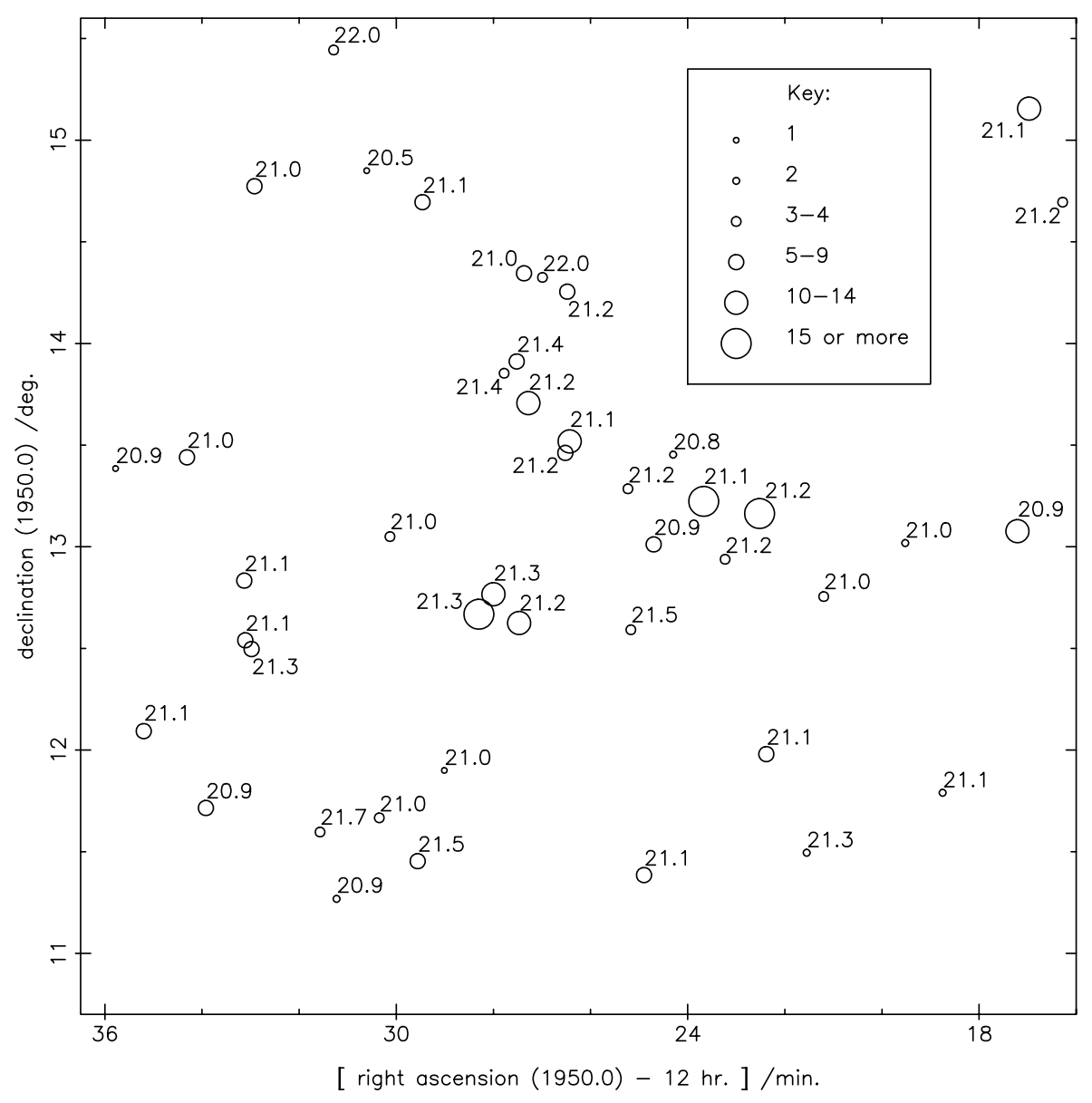

Fig. 3. Sky-brightness estimates in mag $\operatorname{arcsec}^{-2}$ (rounded to the nearest one decimal place) for the positions of galaxy images on Plate U9362. The circle sizes are an indication of the number of measurements used per galaxy (see key)

Table 3. The plate sky-brightness estimates obtained from aperture-photometry measurements in the literature

\begin{tabular}{lcc}
\hline plate & $\begin{array}{c}\text { estimated sky intensity } \\
\left(\text { mag arcsec }^{-2}\right)\end{array}$ & $\begin{array}{c}\text { number of } \\
\text { measurements used }^{\mathrm{b}}\end{array}$ \\
\hline $\mathrm{U} 9362$ & $21.207 \pm 0.016$ & $280 \times U$ \\
$\mathrm{~J} 4882$ & $22.209 \pm 0.025$ & $221 \times B, 221 \times V$ \\
$\mathrm{~J} 9229$ & $21.875 \pm 0.031$ & $239 \times B, 239 \times V$ \\
R2936 & $21.526 \pm 0.040$ & $16 \times R_{\mathrm{C}}$ \\
\hline
\end{tabular}

a The $U$ - and $B_{\mathrm{J}}$-band values listed here were adopted as final zero points but the $R_{\mathrm{C}}$-band value was combined with CCD-derived values (see Sect. 3.4)

b The "number of measurements used" is the number of aperture-photometry measurements on which the corresponding sky estimate is based.

image separately along with totals of the number of measurements used for that image. A separate plot was then generated for each plate (see Fig. 3 for an example) so that the degree of flatness of the sky could be assessed. Once it had been established that the skies were flat (i.e. field effects and vignetting had been adequately compensated for) a grand weighted mean (again in intensity space) was computed for each plate for all of the annuli considered for that plate. These means are listed in Table 3. The annuli were weighted according to the number of intensity units they spanned. Note that the actual standard deviation on the mean for Plate R2936 was 0.020 mag $\operatorname{arcsec}^{-2}$, but it was felt that a value of 0.040 mag $\operatorname{arcsec}^{-2}$ would be more appropriate because this sky estimate was dominated by a single observer's photometry (that of Poulain 1988) which could be expected to be more self-consistent than the same number of measurements made by as many observers.

In the case of Plate R2936, additional zero-point estimates were derived from independently calibrated CCD observations of NGC 4551 and NGC 4478 (see Sect. 3.4). The final zero point adopted for this plate was the weighted mean of the values given in Tables 3 and 4 ; the value of which turned out to be $21.562 \pm$ $0.024 \mathrm{mag} \operatorname{arcsec}^{-2}$. 


\section{4. $R_{\mathrm{C}}$-band CCD photometry}

Whilst it was possible to calibrate the $U$ and $B_{\mathrm{J}}$ plates solely with galaxy aperture photometry measurements obtained from the literature, a different approach was necessary for Plate R2936, owing to the scarcity of published $R$-band, and in particular $R_{\mathrm{C}}$ (Cousins $R$ ) photometry. In this subsection we describe the reduction of $R_{\mathrm{C}}$-band CCD frames of NGC 4478 and NGC 4551 to radial integratedlight profiles that could be used to improve the absolute calibration. The sky-brightness estimates that were obtained from these profiles were used to supplement those estimates obtained using the published aperture photometry described in Sect. 3.3.

Our CCD frames originated from two different sources. We obtained one usable Jacobus Kapteyn Telescope (JKT) CCD frame of NGC 4551 exposed for $300 \mathrm{~s}$ together with frames of flat fields and Landolt (1983) equatorial standard stars from the data archives of the Royal Greenwich Observatory. These observations were made by Peletier and Huizinga over the period 1987 May 22-26. Three additional KPNO 2.1-m telescope CCD frames were kindly provided to us by Reynier Peletier. These latter frames had already been independently calibrated with aperture-photometry measurements corresponding to saturated regions of the galaxy images on the UKST plates (i.e. measurements that could not be used in the calibrations described in Sect. 3.3). Two of the KPNO frames were 300-s exposures of NGC 4551, whilst the third was a 600-s exposure of NGC 4478.

We used essentially the same reduction procedure as described in Young \& Currie (1991) and the same software with only minor modifications. In the case of the JKT frames, the illumination of the flat fields was probably not very uniform (Peletier, private communication) and this may be the origin of the small residual non-linear gradient across the sky that had to be subtracted with a second-degree two-dimensional polynomial function even after flat-fielding process. Despite the difficulties encountered in estimating the sky level beneath the JKT image of NGC 4551 precisely, the probable error on the derived sky surface-brightness value was small enough for this estimate still to be extremely useful. The colour equation we derived from the standard-star frames was:

$$
\begin{aligned}
& R_{\mathrm{C}}= \\
& \quad-2.5 \log _{10}\left(\frac{N_{\star}}{t}\right)-\frac{0.110}{\cos (z)}-0.068(B-V)+21.69
\end{aligned}
$$

in which $N_{\star}$ represents the total number of counts due to the star, $t$ is the exposure time in s and $z$ is the zenith angle. This enabled us to generate a radial integratedmagnitude profile adopting $(B-V)=0.96$ from the Third Reference Catalog of Bright Galaxies of de Vaucouleurs et al. (1991) (RC3).
In the cases of the KPNO 2.1-m telescope frames, after cleaning them, we subtracted the backgrounds (adopting the same background count and sky surface-brightness values as quoted in Peletier et al. 1990) in order to generate radial integrated-magnitude profiles.

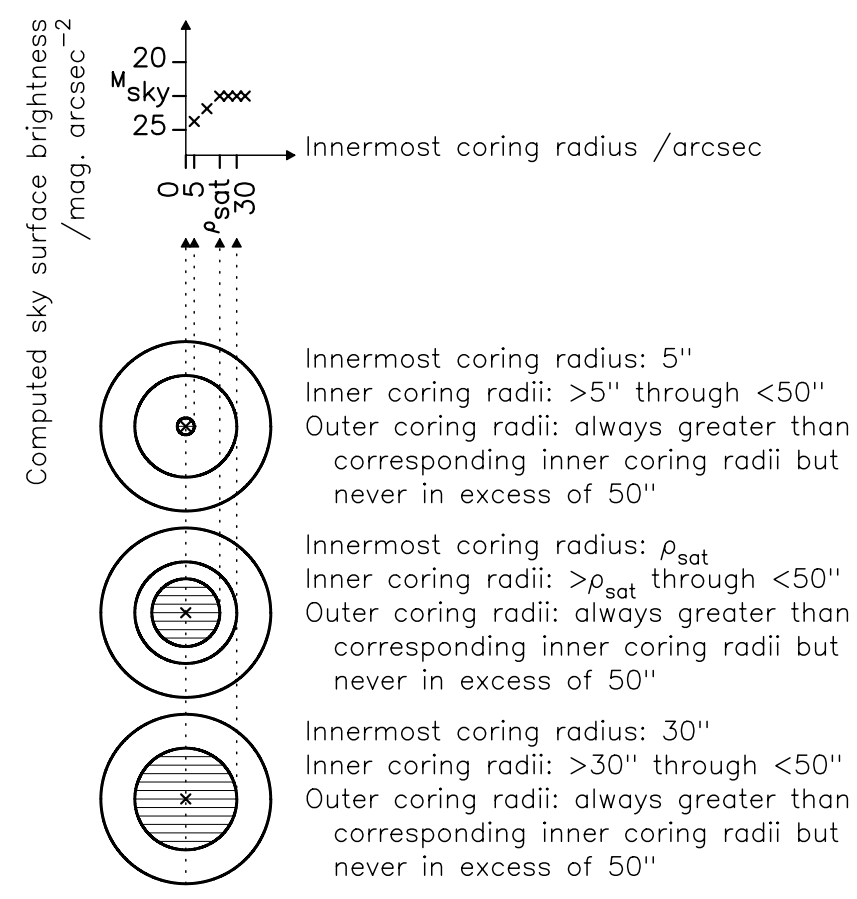

$\rho_{\text {sat }}$ : Radial extent of saturated core region on plate image $M_{\text {sky }}$ : Surface brightness of sky

Extent of central image area excluded from coring

Fig. 4. Diagrammatic representation of the "coring" procedure adopted. An idealised graph of computed sky-brightness versus innermost coring-radius is shown. See Fig. 5 for an example of one of the curves derived experimentally

Table 4. The sky-brightness estimates for Plate R2936 that were derived from CCD observations

\begin{tabular}{lcc}
\hline telescope & galaxy [frame] & $\begin{array}{c}\text { estimated sky brightness } \\
\left(\mathrm{mag} \operatorname{arcsec}^{-2}\right)\end{array}$ \\
\hline JKT & NGC 4551 & $21.56 \pm 0.04$ \\
KPNO 2.1 m & NGC 4551 [1] & $21.55 \pm 0.08$ \\
KPNO 2.1 m & NGC 4551 [2] & $21.62 \pm 0.08$ \\
KPNO 2.1 m & NGC 4478 & $21.65 \pm 0.08$ \\
\hline
\end{tabular}

A coring procedure as described in Sect. 3.3 was necessary in order to estimate sky-brightness values for the positions of NGC 4478 and NGC 4551 on Plate R2936. This time however, CCD radial integrated-magnitude data were used in place of aperture-photometry measurements. Also, in order to estimate the radial extent of core 


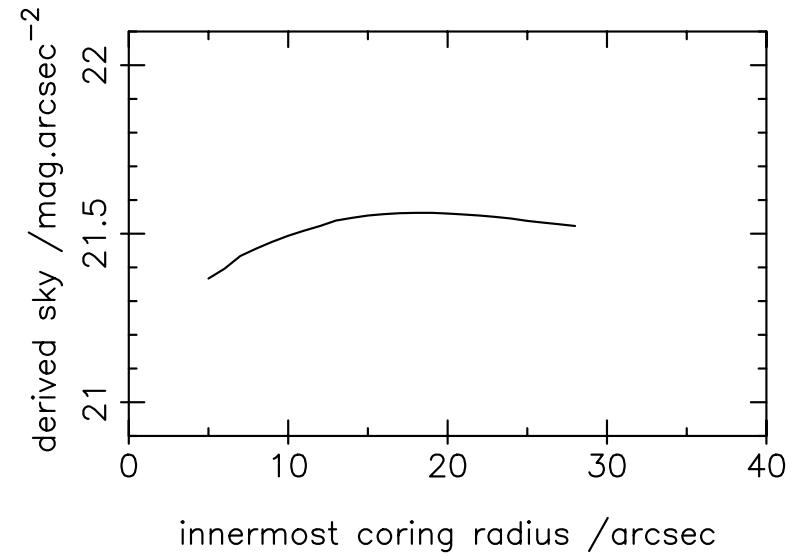

Fig. 5. Computed sky brightness versus innermost coring radius for the R2936 image of NGC 4551 combined with the JKT profile for that galaxy. The minor departure from the ideal curve (which should level off at large radial distances) is presumably due to slight inadequacies in the adopted background functions

saturation, $\rho_{\text {sat }}$, within the plate images, the radius of the central area excluded from the coring was varied. An ideal graph of the variation of estimated sky brightness against innermost coring radius is depicted in Fig. 4. Four such curves were generated (one for NGC 4478 and three for NGC 4551). All of these curves were reasonably wellbehaved, exhibiting only slight negative gradients beyond $\rho_{\text {sat }}$; see Fig. 5 for an example. The sky-brightness estimates for the plate could therefore be read off directly from these plots, and the adopted values are listed in Table 4.

Errors in zero points derived from the KPNO frames are likely to be of the order of $0.05 \mathrm{mag}$ but definitely less than $0.10 \mathrm{mag}$ (Peletier, private communication). Considerably smaller errors would normally be expected for zero points derived from the JKT frame as this frame was calibrated with standard-star observations rather than with aperture photometry. However, the flat fielding problems encountered during the JKT reductions reduced the accuracy somewhat. The turnovers in the graphs such as Fig. 5 yielded handles on the likely sizes of those errors due to inadequacies in the background subtraction. These errors were then propagated together with those originating from earlier stages of the reductions; which were computed according to the same procedure as used in Young \& Currie (1991) for the JKT frame, and using a value of $0.07 \mathrm{mag}$ in the case of the KPNO observations. The resulting error estimates are tabulated in Table 4 together with the corresponding sky-brightness estimates.

\subsection{Generation of unsorted-image parameters}

Prior to sorting galaxy images from stellar ones, the platescan data were first smoothed with a centre-weighted 3- square smoothing algorithm. This algorithm applied a Hanning filter using a 3-by-3 kernel. The resulting smoothing function had a full-width half maximum (FWHM) of 2 pixels. Initial sets of image parameters were then generated by means of Jon Godwin's image-recognition and segmentation programme LLLION (see Godwin 1976 and Dixon 1979 for details). The parameters generated for each image at this stage included: isophotal magnitude, reduced radius $r=\sqrt{r_{\text {major }} r_{\text {minor }}}$ of the limiting isophote, mean surface brightness within that limiting isophote, central surface brightness, surface brightness of the brightest overlapping isophote (in cases where nearby images overlapped with the one in question) ellipticity, position angle and the isophote used for the determination of the position angle.

The surface brightness at which saturation effects (due to the limitations in the dynamic ranges of both the emulsion and of the microdensitometer) became significant was estimated for each plate. This was achieved by the assembly of several composite stellar profiles per plate (by the same method as those described in Sect. 3.6 were). By varying the upper surface-brightness limits on the portions of the stellar profiles to be included in the composite profiles, the onset of saturation could be observed. Composite stellar profiles based only on the unsaturated portions of images yielded plots similar to Fig. 6, but at a certain point, the inclusion of brighter isophotes caused some data points [from previously excluded saturated images] to appear below the narrow stellar locus. Once the surface-brightness level at which the onset of saturation occurred had been established for each plate, galaxy images with saturated cores could then be identified and flagged.

Metcalfe et al. (1995) have demonstrated that in previous survey work based on both the COSMOS and APM measuring machines, there is increased scatter in [both isophotal and total] galaxy magnitudes for objects with higher central surface brightnesses. They showed that this effect (which should not be confused with true saturation effects) is almost certainly due to limitations in the machines. They conclude that several precautions ought to be taken to minimise this problem when working with COSMOS or APM galaxy data. All of their concerns have been met during the compilation of the VPC.

Except in the $R_{\mathrm{C}}$ band, between forty and fifty calibrating galaxies of widely varying central surface brightnesses have been used per plate, enabling us to fix our $U$ and $B_{\mathrm{J}}$ zero points with confidence. All of the plates were taken under conditions of poor to very poor seeing, which should reduce the possibility of scale errors in our magnitude scales. Also, the brightest galaxy images with unsaturated VPC photometry tend to correspond to objects that are of relatively low surface brightness (which abound in the direction of the Virgo Cluster). These images tend not to have profiles that fall off very steeply near their centroids, and are thus very much less susceptible to the effects discussed by Metcalfe et al. It should also be 
mentioned that even if some minor [true] saturation effects were present in the VPC's [integrated] isophotal magnitude measurements, they would be unlikely to have any significant effect on the VPC's $t$-system total magnitudes (see Sect. 4). This is because in the $t$ system the central surface brightness is in fact an extrapolated quantity.

Table 5. The isophote limits adopted during the image segmentation and the relevant seeing conditions

\begin{tabular}{|c|c|c|c|}
\hline plate & $\begin{array}{c}\text { faint limit } \\
\left(\mathrm{mag} \operatorname{arcsec}^{-2}\right)\end{array}$ & $\begin{array}{c}\text { bright limit } \\
\left(\mathrm{mag} \mathrm{arcsec}^{-2}\right)\end{array}$ & $\begin{array}{l}\text { seeing FWHM } \\
\quad(\operatorname{arcsec})\end{array}$ \\
\hline U9362 & 25.0 & 20.9 & 2.7 \\
\hline J4882 & 25.0 & 21.9 & 3.0 \\
\hline J9229 & 25.0 & 21.9 & 1.7 \\
\hline R2936 & 24.0 & 20.9 & $\sim 3.5$ \\
\hline
\end{tabular}

The faintest usable limiting isophotes did of course vary from plate to plate, even between plates exposed in the same band (on account of differences in the levels of plate noise and quantization of the intensity levels) though for consistency's sake the same limiting isophote of $25.0 \mathrm{mag} \operatorname{arcsec}^{-2}$ was adopted for both $B_{\mathrm{J}}$ plates. Although it had been decided to obtain as complete a galaxy sample as possible down to the integrated isophotal magnitude of $B_{\mathrm{J}_{25.0}} \leq 18.5$ all images down to $B_{\mathrm{J}_{25.0}}$ $\leq 20.0, U_{25.0} \leq 20.5$ and $R_{24.0} \leq 20.0$ were segmented in anticipation of differences (both systematic and random) between parameters derived from the different plates. Details of the isophote limits adopted are listed for all four plates in Table 5. Note that although Plate J9229 was made under better seeing conditions than Plate J4882 was (which would normally yield plate scans of smaller dynamic range), Plate J4882 was quite a dark plate and was not nitrogen flushed. As a result, the dynamic ranges of the plate-scan data from these two plates were actually found to be very similar.

\subsection{Star-galaxy sorting}

Although the tasks of star-galaxy sorting and image selection are often still performed wholly by eye (the entries in the VCC, for example, were selected by visual inspection alone) many different automated techniques have been developed for discriminating between images of stars and galaxies ever since those of Oemler (1974), Godwin (1976) and MacGillivray et al. (1976). These methods all involve isolating images that approximate well to the point spread function and are therefore probably stellar, from those with shallower profiles that are probably not stellar; though it should be noted that none of these automated procedures can be relied upon to yield a pure galaxy sample.

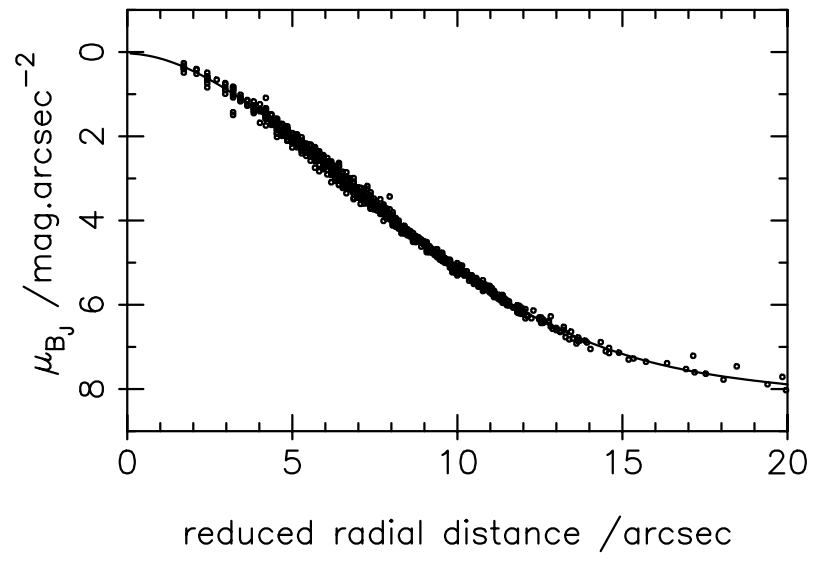

Fig. 6. The composite stellar profile from plate J4882 on which the star-galaxy sorting was based. The best-fitting eighth-degree polynomial function (solid line on graph) was adopted as the template for stellar images

Godwin's method which was followed here assumes that the shape of a stellar image profile is essentially independent of apparent magnitude except of course when saturation effects are at play. A separate set of composite stellar-image profiles was assembled for each plate by sliding over a hundred stellar profiles (from stars of widely different apparent magnitude) onto one another in log surface-brightness space, omitting only those isophotes brighter than the saturation limit. Whilst a composite profile was used to quantify the point-spread function for each smoothed plate-scan, a single composite [and unsaturated] J4882 profile was selected for use as a template in the sorting procedure; see Fig. 6. As the original intention was to generate a $B_{\mathrm{J}_{25}}$-limited dataset, a $B_{\mathrm{J}}$ plate had to be used for the sorting. It was decided to use the J4882 plate because upon initial inspection of galaxies in Field A it appeared that this plate was slightly deeper than Plate J9229. This turned out to be a rather unfortunate choice because J4882 was later discovered to be the inferior plate of the two by quite a considerable margin.

Carter \& Godwin (1979) introduced the image-profile parameter, $\alpha$. This was defined as the slope of the linear regression of the logarithmic surface-brightness residuals against $\log$ radius. It was evaluated after first minimising the sum of the residuals, by sliding the image profile onto the adopted template in negative log surface-brightness space. Stellar images tend to have $\alpha$ in the vicinity of zero. As galaxies tend to have shallower profiles, and the residuals were evaluated in the sense: image minus template, their $\alpha$ values tend to be negative. At very faint magnitudes however, the separation between stars and galaxies becomes less pronounced. This is because fainter galaxy images approximate more closely to the stellar template and the profiles of both stars and galaxies get noisier as the images get fainter. This effect can be seen in Fig. 7 .

It was decided to inspect visually all images with both $\alpha \leq-0.065$ and $B_{\mathrm{J}_{25}} \leq 19.0$ on plate $\mathrm{J} 4882$ bearing in 


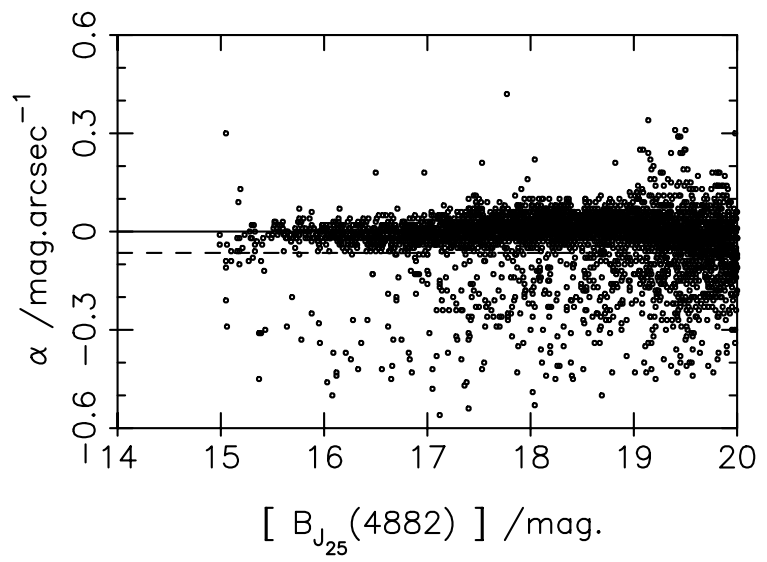

Fig. 7. The slope parameter, $\alpha$, plotted as function of $B_{\mathrm{J}_{25}}$ magnitude for all images within Field $B$ (one quarter of the total area scanned) for which it could be evaluated. All images of $B_{\mathrm{J}_{25}} \leq 19.0$ lying below the dashed line (representing $\alpha=-0.065$ ) were inspected visually as part of the star-galaxy separation procedure

mind that galaxies with steeper profiles almost invariably had very starlike appearances [on these Schmidt plates at least] and would probably escape an independent visual selection process anyway. A glass copy of plate J9229 was used in conjunction with a light table and binocular microscope (of maximum magnification 25 times) for this purpose. The total number of images that were visually inspected was almost 4000; and of these roughly one third were confirmed to be galaxies. Stellar contamination of the galaxy sample is almost certainly less than $0.25 \%$ (i.e. less than three objects) as only very occasionally was there any doubt as to whether an object that satisfied the automatic galaxy-candidate selection process was a star or a galaxy. The number of galaxies with $\alpha$ s in excess of -0.065 (and therefore omitted from the final catalogue) is not certain. Nevertheless this number is expected to be very small for galaxies brighter than the isophotal-magnitude completeness limit (see Sect. 6.2).

\subsection{Generation of sorted-image parameters}

Equatorial coordinates were generated from the plate $(x, y)$ system by means of the Starlink package ASTROM. 25 reference stars of $m_{B}>10.0$ were selected from Heckmann et al.'s (1975) AGK3 astrometric catalogue for this coordinate conversion.

Those galaxy images that were unsaturated on Plate J4882 and which were successfully resolved from all adjacent images during image segmentation, of which there were over a thousand, were matched with their counterparts on the other plates by means of $3 \times 2$-matrix transformations in their plate $(x, y)$ coordinates (i.e. allowing for both shift and rotation). The colour-generating programme KOLORE (see Godwin 1976 and Dixon 1979) was then run to compute $\left(U-B_{\mathrm{J}}\right)$ and $\left(B_{\mathrm{J}}-R_{\mathrm{C}}\right)$ equal-area colours together with corresponding reduced radii, position angles, ellipticities and isophote information. Means were then taken of parameters derived independently from each of the two $B_{\mathrm{J}}$ plates, assigning equal weights to the plates. At a later stage however, it was discovered that those parameters generated from Fields $\mathrm{C}$ and D of plate J4882 were unreliable due to inadequate hypersensitisation of those regions. It was therefore decided only to use those parameters derived from plate J9229 for those two fields. Also, when an image was affected by a plate flaw, only the scan data from the unaffected plates were used, resulting in the loss of some information. Luckily though, only a handful of images was affected. Note that total colours were also generated at a later stage (as described in Sect. 4.2).

It was not possible to derive reliable image parameters for saturated objects owing to the loss of information about the image cores. However, as many of the saturated objects are amongst the most prominent galaxies in the Virgo field, the majority were already catalogued in the VCC and/or the RC3, whence certain parameters could be extracted. Some of the objects were not in either catalogue however, and eye estimates of their apparent $B_{\mathrm{J}_{25}}$ magnitudes were necessary. In such cases the values arising from the segmented plate scans could only be used as a faint limit, as saturation effects would inevitably cause the image brightnesses to be underestimated.

Certain galaxy images were contaminated by adjacent stellar images and/or by other galaxies. The imagerecognition and segmentation programme LLLION was able to separate most overlapping images from one another. However, in 51 cases LLLION could not resolve them cleanly, because the degree of overlap was so large and/or the disparity in brightness between the objects was so great. Under such circumstances, apparent $B_{\mathrm{J}_{25}}$ magnitude estimates were based on the values derived from the image segmentation for the unresolved objects combined, minus an eye-estimated value for the contaminating object. When the contaminating object was a star, the magnitude of the star was estimated by finding by eye an isolated stellar image of similar size and looking up its magnitude in the original unsorted list of segmented objects. Subtracting contaminating-galaxy images was a less precise affair, and no attempt was made to separate the closest pairs of double galaxies, unless the segmentation algorithm had already succeeded to a certain extent.

\subsection{Data for the saturated images}

Those galaxy images that were saturated on the plate scan of J4882 were treated separately. In many cases, right ascensions, declinations, NGC or IC designations, VCC numbers, ellipticities, position angles as well as $(U-B)_{\mathrm{T}}$ colours could be extracted from the RC3. $\left(U-B_{\mathrm{J}}\right)_{\mathrm{B}_{25}}$ colours were derived from the $(U-B)_{\mathrm{T}}$ values by means of Fig. 6b on page 35 of de Vaucouleurs et al. (1976); the 
required $r_{\mathrm{B}_{25}}$ apparent radii values having been obtained from the RC3.

$B_{\mathrm{J}_{25}}$ values were then estimated from those $B_{\mathrm{T}}$ values quoted in the VCC, by subtracting a typical offset of 0.1 mag: $B_{\mathrm{J}_{25}} \approx B_{\mathrm{T}}-0.1$, from $B_{\mathrm{J}_{\mathrm{T}}} \approx B_{\mathrm{T}}-0.3$ and $B_{\mathrm{J}_{25}} \approx B_{\mathrm{J}_{\mathrm{T}}}+0.2$. These equations are only intended as provisional approximations, and are not applicable to dwarfs (which tend not to be saturated in the VPC).

\subsection{Radial velocities and morphological types}

Radial velocities were extracted from the RC3 whenever possible, but original sources had to be consulted for most objects known to be receding at velocities in excess of $15000 \mathrm{~km} \mathrm{~s}^{-1}$, as that was the velocity limit of the RC3 galaxy sample. Morphological types were extracted from the VCC whenever listed therein, but visual inspection of the glass copy plate was necessary for those objects with $B_{\mathrm{J}_{25}} \leq 17.0$ not catalogued in the VCC. It was decided not to attempt the morphological typing of galaxies fainter than $B_{\mathrm{J}_{25}}=17.0$, as beyond this limit the galaxy images were generally too small to be typed reliably.

\section{Total magnitudes}

\subsection{The $t$ system}

A reasonably discerning extrapolation procedure was required for the VPC galaxy sample as (1) most VPC galaxies are too bright and/or extended for their images to be seeing dominated, and (2) a wide variety of morphological types (including of course a very large number of lowsurface-brightness galaxies) are represented. The totalmagnitude system we adopted is the $t$ system of Young (1994) and and Young et al. (submitted); as outlined by Young (in press). This system involves the smoothing of galaxy images prior to modeling them by means of Sérsic's (1968) law. Note that in the specific case of unnucleated ellipticals, prior smoothing is not actually necessary as demonstrated by Young \& Currie (1994) who fitted Sérsic's law to high-resolution images of dwarf and intermediate ellipticals.

\subsection{Total magnitudes and colours for unsaturated images}

In the case of the low-resolution VPC data, Sérsic's law was found to offer an excellent fit to virtually every unsaturated surface-brightness profile, irrespective of morphological type. This can be seen from the thirty example plots presented in Appendix $\mathrm{A}^{2}$. No total magnitude estimates were attempted for saturated galaxies. Instead, Young et al. (in preparation) will present $t$-system total magnitudes for these objects based on even lowerresolution CCD photometry.

\footnotetext{
2 These plots correspond to the first thirty unsaturated galaxies in the VPC, and have therefore been selected on the basis of their right ascensions alone.
}

During the image-segmentation stage of the reductions, profile information was generated for each galaxy image in the form of ellipticities, position angles and reduced radii, for all unsaturated isophotes in $0.25 \mathrm{mag} \operatorname{arcsec}^{-2}$ intervals brightward of the limiting one. In the case of the $B_{\mathrm{J}}$ plates, this corresponded to a maximum of 12 levels (a maximum of 16 in the case of plate U9362 and a maximum of 12 in the case of R2936).

The profile parameterizations were achieved by incrementing $n$ from 0.2 through 3.0 in steps of 0.01 , and attempting to fit a straight line to $\mu(r)$ as a function of $r^{n}$. Points corresponding to isophotes that overlapped with those of other images (as represented by five-pointed stars in Appendix A) were assigned weights $50 \%$ lower than those assigned to the remaining points (as represented by asterisks in Appendix A). $\chi^{2}$ was evaluated for each fit, and the parameters $\left(n, r_{0}\right.$ and $\left.\mu_{0}\right)$ that provided the best fit for a particular galaxy, were used to derive a totalmagnitude estimate for that galaxy by means of Young \& Currie's (1994) Eq. (3).

In the case of the $B_{\mathrm{J}}$ band, although we had only parametrised the profiles from Plate J9229, as we had already computed two usable $B_{J_{25}}$ values for those galaxies lying within Fields $\mathrm{A}$ and $\mathrm{B}$ (one value from each $B_{\mathrm{J}}$ plate), we were still able to average the extrapolated total magnitude values. This was achieved by subtracting $\left[B_{\mathrm{J}_{25}}(J 9229)-B_{\mathrm{J}_{25}}(J 4882)\right] / 2$ from the total magnitudes derived from the parametrizations of the Plate J9229 images.

The transformation of total magnitudes from the $B_{\mathrm{J}}$ system to the $B$ system was achieved by means of Eq. (1), and the approximate relationship:

$$
\left(B_{\mathrm{J}}-R_{\mathrm{C}}\right)=0.46( \pm 0.10)(B-V)+0.83( \pm 0.07),
$$

which was derived from Fig. 8; a two-colour plot for unsaturated VPC galaxies with $(B-V)$ colours previously measured by Caldwell (1983) Gallagher \& Hunter (1986) Bothun et al. (1986) and/or Drinkwater \& Hardy (1991). Note that although the $\left(B_{\mathrm{J}}-R_{\mathrm{C}}\right)$ and $(B-V)$ colour measurements were not based on identical regions of each galaxy concerned, errors due to colour-gradients are expected to be considerably smaller than those due to the inherent scatter in such a colour-colour plot.

\subsection{VPC magnitudes already published}

The total magnitudes listed in Table 4 of Young \& Currie (1995) as well as those VPC magnitude values listed in Table 9 of Drinkwater et al. (1996), were extracted from an earlier version of the VPC; that of Young (1994). The magnitudes were computed by a variant on the method described in Sect. 4.2. This method involved adding the integral of the same function between the isophotal radius $r=r_{25}$ and $r_{25}<<r<<\infty$ to the linear-unit equivalent of the $B_{J_{25}}$ value tabulated in the VPC for each galaxy. The integral was evaluated numerically using the 


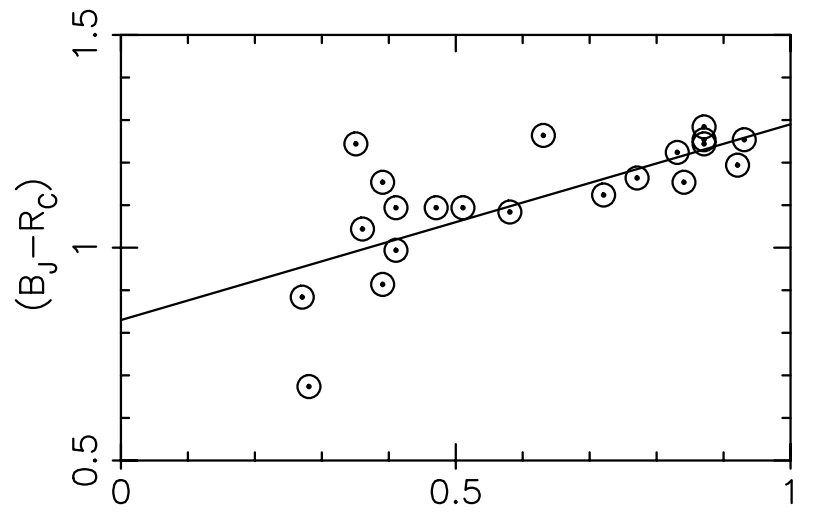

photoelectric aperture $(B-V)$

Fig. 8. Unsaturated VPC galaxies for which $(B-V)$ measurements have already been published (see text for references). This two-colour plot was used to derive a transformation equation between $\left(B_{\mathrm{J}}-R_{\mathrm{C}}\right)$ equal-area colours and $(B-V)$ by means of the best-fitting least-squares (on $\left(B_{\mathrm{J}}-R_{\mathrm{C}}\right)$ because the $(B-V)$ values are expected to be more accurate as they are photoelectric measurements rather than photographic ones) straight line

Compound Form of Simpson's Rule, with 2000 intervals spanning the range $r=r_{25}$ to the point at which $\mu(r)$ had dropped to $\mu_{0}+(10 \times 1.086) \mathrm{mag}_{\operatorname{arcsec}}{ }^{-2}$ :

$$
\begin{aligned}
& 2 \pi \int_{r_{25}}^{10^{\frac{1}{n}}} \sigma r \mathrm{~d} r= \\
& \quad \frac{\pi \sigma_{0}\left(10^{\frac{1}{n}} r_{0}-r_{25}\right)}{3000}\left[f_{0}+4\left(f_{1}+f_{3}+\ldots\right.\right. \\
& \left.\left.\quad+f_{1999}\right)+2\left(f_{2}+f_{4}+\ldots f_{1998}\right)+f_{2000}\right]
\end{aligned}
$$

where $f_{0}=r \sigma\left(r=r_{25}\right)$ and $f_{2000}=r \sigma\left(r=10^{\frac{1}{n}} r_{0}\right)$. It was adopted at a time when the catalogue listed $\overline{B_{\mathrm{J}_{25}}}=\left[B_{\mathrm{J}_{25}}(J 9229)+B_{\mathrm{J}_{25}}(J 4882)\right] / 2$ and $\sigma_{B_{\mathrm{J}_{25}}}=$ $\left|\left[B_{\mathrm{J}_{25}}(J 9229)-B_{\mathrm{J}_{25}}(J 4882)\right] / 2\right|$ values for Field A and B objects; but did not list the senses of the differences between the $B_{\mathrm{J}_{25}}(J 9229)$ and $B_{\mathrm{J}_{25}}(J 4882)$ values. With this method, it was therefore still possible to obtain averaged total magnitudes based on the isophotal magnitudes from two plates [together with extrapolations from one plate] without knowing the senses of the differences between the plates.

In a small minority of cases there was a significant difference between the sizes of the extrapolations obtained by the different methods, but on average the choice of method made little difference. The mean extrapolation [for the VPC as a whole] beyond $\overline{B_{\mathrm{J}_{25}}}$ due to the old method was $0.348 \mathrm{mag}$, compared to a mean of 0.352 for the more rigorous method used in this paper; though these mean extrapolations were 0.670 and 0.696 respectively for the dwarf elliptical subsample of 64 objects presented in Young \& Currie (1995). The systematic difference between the two sets of magnitude values is due to the minority of objects for which the light contribution from regions exterior to $r=10 \frac{1}{n} r_{0}$ would be significant should the profiles be extrapolated to $r=\infty$.

\section{The dataset}

Appendix A contains examples of the $B_{\mathrm{J}}$-band surface photometry obtained from Plate J9229. The profiles correspond to those thirty unsaturated objects of lowest right acension.

The complete dataset can be divided into three parts: Appendix B, which contains the surface photometry obtained from each plate separately for unsaturated objects that were not inextricably merged with adjacent objects (but excluding Fields A and B in the case of Plate J4882); Appendix C, which contains the main catalogue (of galaxies VPC 1 through 1129); and Appendix D, the extension to the main catalogue (which is reserved for those 51 objects whose images were severely contaminated by overlapping stellar or galaxy images, these being designated VPCX 1 through 51). Machine-readable versions of Appendix B (in ASCII format) and Appendix C (in both ASCII and FITS formats) are available from the CDS.

In addition to the complete dataset, we have included two more appendices for the convenience of readers. The first of these is Appendix E, which contains a summary catalogue of key parameters in tabular from. This is available from the CDS in electronic form as a $\mathrm{IAT}_{\mathrm{E}} \mathrm{Xfile}$. The last appendix is Appendix F, which contains a list of VPC objects with NGC or IC designations.

The parameters listed in Appendix E are:

Column 1: VPC designation, galaxies being numbered in order of increasing right ascension (1950.0), objects at identical right ascensions being numbered in order of increasing declination (1950.0), those objects saturated in $B_{\mathrm{J}}$ being flagged with an asterisk;

Column 2: right ascension minus 12 hours (1950.0) in minutes and seconds of time; followed by declination (1950.0) in degrees, minutes and seconds of arc (mean error of $1 . " 5$ on each coordinate);

Column 3: VCC designation and cluster-membership assignment from Binggeli et al. (1985), their assignments being denoted "B" for background, "P" for possible member and "M" for member;

Column 4: morphological type if discernable from visual inspection of a copy plate of Plate J9229 and/or a VCC galaxy: "E" for elliptical, "S" for spiral, "S0" for lenticular, "I" for irregular, "BCD" for blue compact dwarf, "pre" for pre-merger and "pec" for peculiar (objects that defied classification being assigned "?"); followed by a one digit coding for the source of the morphological classification adopted: [1] for the VCC and [2] for this work; note that we have not flagged dwarf ellipticals because there may well be a continuous range of galaxies separating "true" classicals from "true" dwarfs; 
Column 5: apparent $B_{\mathrm{J}}$ magnitude to the $25.0 \mathrm{mag} \operatorname{arcsec}^{-2}$ isophote; the value quoted being the mean $^{3}$ of two values independently derived from Plates J4882 and J9229 (estimated mean error of $\sim 0.1 \mathrm{mag}$ ), except when followed by a single digit within the range [1] through [9] which denotes an alternative source of $B_{\mathrm{J}_{25}}$ estimate or measurement: [1] for de Vaucouleurs \& Pence (1979) Table 2, [2] for de Vaucouleurs \& Pence (1979) Table 4, [3] for Binggeli et al. (1984), [4] for Binggeli et al. (1985), [5] for Karachentsev \& Karachentseva (1982), [6] for the mean of sources [1] and [4], [7] for the mean of sources [2] and [4], [8] for the mean of sources [4] and [5], and [9] this work when an estimate was made by the authors by eye;

Column 6: standard error on the mean $B_{\mathrm{J}_{25}}$ value when applicable; followed, only when flagged in Col. 5 by a code number other than [9], by a single character "R" for reliable or "U" for unreliable from the VCC;

Column 7: $B_{\mathrm{J}_{\mathrm{t}}}$ [total extrapolated] magnitude, derived according to the procedure described in Sect. 4.2 (estimated mean error of $\sim 0.15 \mathrm{mag}$ );

Column 8: transformed $B_{\mathrm{t}}$ [total extrapolated] magnitude, derived from $B_{\mathrm{J}_{\mathrm{t}}}$ via Eqs. (1) and (3) (estimated mean error of $<0.20 \mathrm{mag}$ );

Columns 9, 10 and 11: $B_{\mathrm{J}}$-band Sérsic surface-brightness parameters: $n$ (profile shape parameter), $\mu_{0}$ (extrapolated central surface brightness $/$ mag $\operatorname{arcsec}^{-2}$ ) and $r_{0}$ (scale length parameter) respectively from Plate J9229 for galaxies unsaturated in $B_{\mathrm{J}}$, all derived according to the procedure described in Sect. 4.2;

Column 12: $\left(U-B_{\mathrm{J}}\right)$ equal-area colour if relevant VPC photometry unsaturated and not heavily merged (estimated mean error of $\sim 0.2 \mathrm{mag})$, or $(U-0.65 B-0.35 \mathrm{~V})_{\mathrm{T}}$ from the RC3 whenever possible if VPC photometry saturated;

Column 13: $\left(B_{\mathrm{J}}-R_{\mathrm{C}}\right)$ equal-area colour if relevant VPC photometry unsaturated and not heavily merged (estimated mean error of $\sim 0.2 \mathrm{mag}$ );

Column 14: heliocentric radial velocity in $\mathrm{km} \mathrm{s}^{-1}$ (based on the approximation $v=c z$, where $v$ is approximate velocity, $c$ is the speed of light in a vacuum and $z$ is redshift); followed by a one digit code for the source of the velocity measurement: [1] for Karachentsev \& Karachentseva (1982), [2] for Karachentsev et al. (1983), [3] for Binggeli et al. (1985), [4] for Rhee \& Katgert (1988), [5] for de Vaucouleurs et al. (1991), [6] for Binggeli et al. (1993), and [7] for Drinkwater et al. (1996); when more than one velocity measurement was available for a particular galaxy, the value that was adopted was either the value with the smallest associated error if the measurements were in broad agreement, or the value from the more recent com-

\footnotetext{
3 The mean values being applicable to Virgo Fields A and B only. Due to significant de-sensitisation (as described in Sect. 7.1) of Fields C and D on Plate J4882, only Plate J9229 was used for objects lying in these two latter fields.
}

pilation if the measurements were not in broad agreement, i.e. [7] supersedes [6], and [6] supersedes earlier compilations such as [5] (quoted errors vary greatly from source to source but are typically of the order of $50 \mathrm{~km} \mathrm{~s}^{-1}$ ).

The VPC also contains: $U_{25}$ and $R_{\mathrm{C}_{24}}$ isophotal magnitudes together with corresponding apparent angular radii, mean surface-brightnesses within the limiting isophotes, measured central surface brightnesses, brightest overlapping isophotes with adjacent images, $\left(U-B_{\mathrm{J}}\right)$ and $\left(B_{\mathrm{J}}-\right.$ $R_{\mathrm{C}}$ ) total colours, ellipticities, position angles, and the errors associated with various parameters (including radial velocity measurements). However, these parameters are confined to the the full version of the main catalogue.

\section{Scope of the catalogue}

Previous catalogues of Virgo Cluster galaxies have been limited in several respects. First, the vast majority of their galaxy magnitudes were estimated by eye without independent zero-point calibrations, secondly, the galaxy samples themselves were selected by eye and thirdly, they were confined to a single pass band. The VPC does not suffer from any of these limitations. Also, the VPC does not discriminate against background galaxies (unlike the VCC which excludes non-Zwicky objects that were deemed to be in the background).

The VPC however, covers a region of sky smaller in angular extent than those covered by de Vaucouleurs \& Pence (1979) or by the VCC. The VPC survey area, which is centred on Cluster A, is compared with the VCC's in Fig. 9.

\subsection{Galaxy magnitudes}

Most VPC-galaxy magnitudes have been derived by the numerical integration of segmented plate-scan data and have been calibrated with several CCD frames and/or several hundred aperture-photometry measurements. We therefore expect these magnitudes to be a significant improvement on those found in previous catalogues. For the highest surface-brightness VPC galaxies however, the plate-scan data suffered from saturation effects, and alternative measurements had to be sought from the literature. As can be seen from Fig. 10, saturation was a problem in the $B_{\mathrm{J}}$ band for most galaxies brighter than $B_{\mathrm{J}_{25}}=15.5$, though fewer galaxies were saturated in the $U$ band. For a very small minority of saturated galaxies, no previous measurements could be found in the literature and eye estimates were necessary.

\subsection{The galaxy sample}

There has been much discussion concerning the completeness of galaxy surveys in the literature, and particularly of those conducted by eye; see e.g. Phillipps et al. (1988). As the VPC galaxy sample was selected primarily by means of an automated process, it was possible to quantify the 


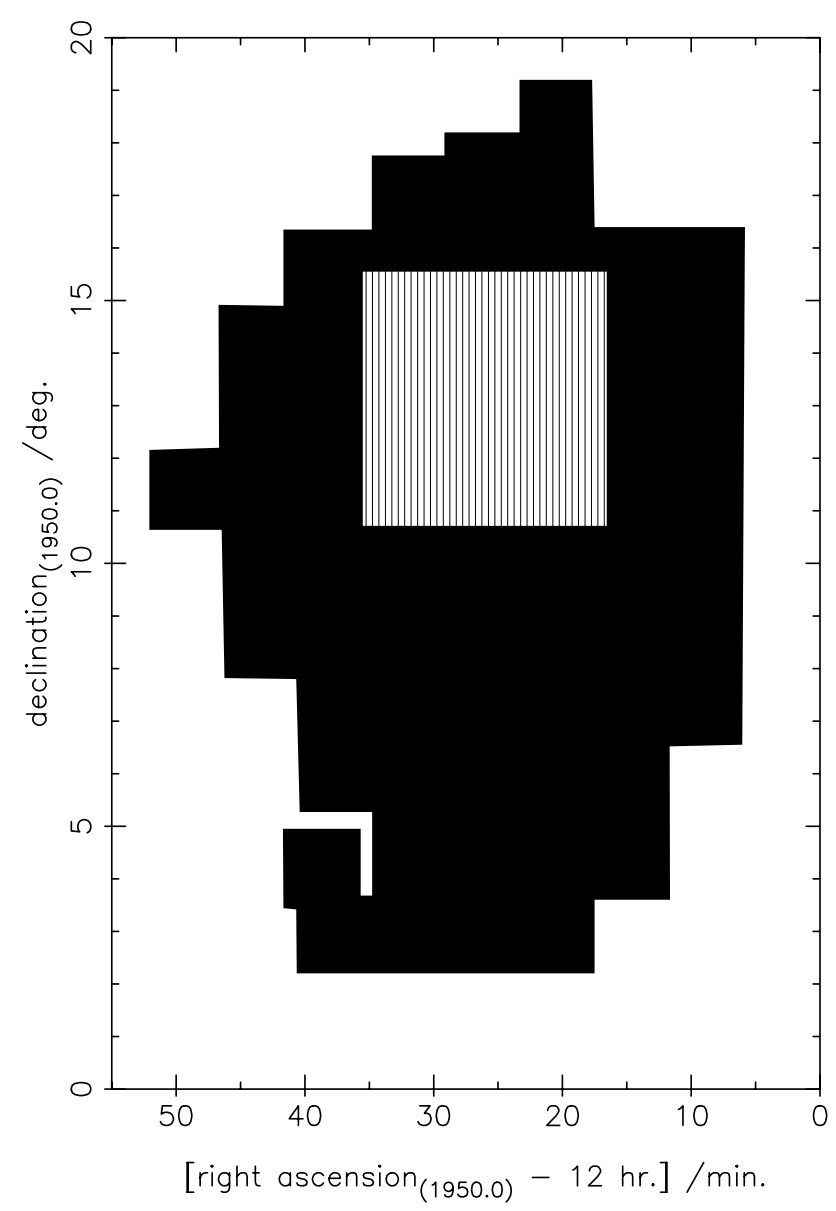

Fig. 9. Map of the areas of the sky surveyed by the VPC (vertically shaded area) and VCC (heavily shaded area $\cup$ VPC survey area)

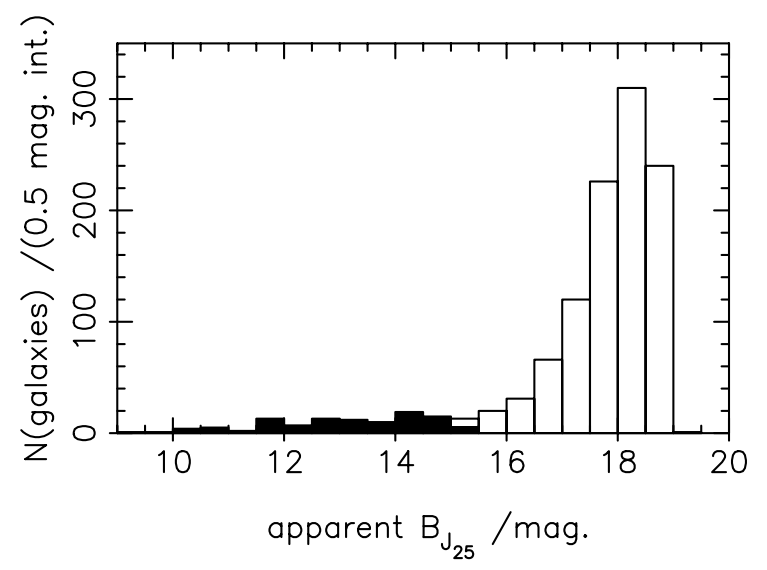

Fig. 10. Frequency distribution of VPC galaxies as a function of $B_{\mathrm{J}_{25}}$. The heavily shaded areas represent objects saturated in $B_{\mathrm{J}}$ observational limits to the sample with reasonable precision. These limits were:

(1) $B_{\mathrm{J}_{25}}(J 4882) \leq 19.0$ for the whole survey area and

(2) profile-slope parameter (see Sect. 3.6) $\alpha \leq-0.065$.

However, Plate J4882 was found to be significantly desensitised over Fields $\mathrm{C}$ and $\mathrm{D}$ (by $\sim 0.3 \mathrm{mag}$ ) and the magnitudes for those fields had therefore to be based on J9229 alone. Mean magnitudes (based on both the J4882 and J9229 values) were nevertheless adopted for Fields A and $\mathrm{B}$. These considerations were found to lead to the VPC-sample completeness limits of:

(1a) $B_{J_{25}} \leq 18.5$ for Fields A and B, or

(1b) $B_{\mathrm{J}_{25}} \leq 18.0$ for Fields C and D, and

(2) profile-slope parameter $\alpha \leq-0.065$,

which are derived at the end of this subsection.

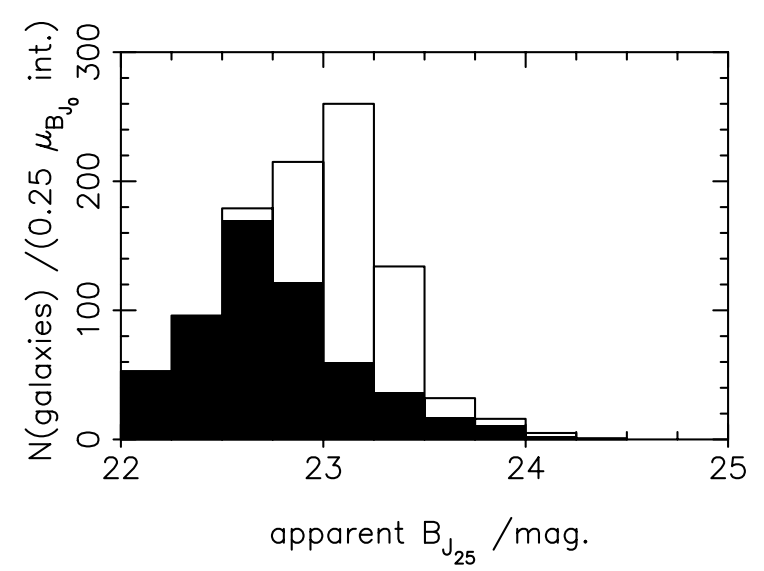

Fig. 11. Frequency distribution of VPC galaxies unsaturated in $B_{\mathrm{J}}$, as a function of peak surface brightness; the heavily shaded areas representing those galaxies brighter than the completeness limits $\left(B_{\mathrm{J}_{25}}=18.5\right.$ for Fields $\mathrm{A}$ and $\mathrm{B} ; B_{\mathrm{J}_{25}}=18.0$ for Fields C and D)

Should populations of ultra-low-surface-brightness galaxies exist (of peak surface brightnesses fainter than $25.0 \mathrm{mag} \mathrm{arcsec}^{-2}$ in $B_{\mathrm{J}}$ ) they would therefore remain uncatalogued regardless of their apparent magnitude because isophotal magnitudes rather than total magnitudes were used to select the galaxy sample. Figure 11 would however suggest that galaxies with peak surface brightnesses fainter than $\sim 24.0 \mathrm{mag} \operatorname{arcsec}^{-2}$ do not normally have flat enough surface-brightness profiles to qualify as 18th-magnitude (or brighter) objects, unless they possess very extensive haloes of surface brightness slightly fainter than $25.0 \mathrm{mag} \operatorname{arcsec}^{-2}$. Likewise, discrimination against ultra-low-surface-brightness galaxies on account of using isophotal magnitudes cannot be a problem unless such galaxies have very flat profiles and are quite large in angular extent (e.g. an object of mean surface brightness 
$=26.00 \mathrm{mag} \operatorname{arcsec}^{-2}$ would have to subtend 28 arcsec in diameter in order to be a 19.0 magnitude object or 35 arcsec in diameter in order to be an 18.5 magnitude object). Although giant galaxies of very low surface brightness are known to exist (e.g. Malin 1, which escaped detection in the VPC) it is unlikely that such objects could be prolific enough to be a major hazard.

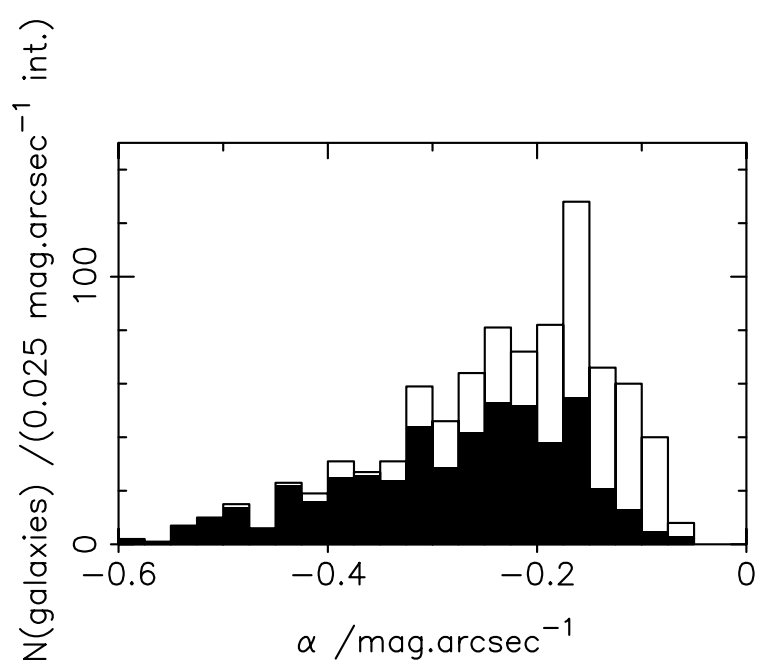

Fig. 12. Frequency distribution of VPC galaxies as a function of the profile-slope parameter $\alpha$ (see Sect. 3.6); the heavily shaded areas representing those galaxies brighter than the completeness limits of $\left(B_{\mathrm{J}_{25}}=18.0\right.$ for Fields $\mathrm{A}$ and $\mathrm{B} ; B_{\mathrm{J}_{25}}=18.5$ for Fields C and D)

It would appear from Fig. 12 that brighter than the completeness limit of $B_{\mathrm{J}_{25}}=18.0$ galaxy loss due to the limit $\alpha \leq-0.065$ is probably not significant. However, from the same figure it can be seen that [due to this criterion alone] the number of galaxies lost from the sample within the range $18.0<B_{\mathrm{J}_{25}}<19.0$ is probably quite significant. In other words, for $B_{J_{25}}>18.0$, the galaxy profiles tend to be significantly more starlike than for $B_{\mathrm{J}_{25}}<18.0$. Unfortunately, it is rather difficult to extrapolate the frequency distribution of galaxies as a function of $\alpha$ beyond the $\alpha=-0.065$ cut off with great confidence, even though the indications are that the frequency is already in sharp decline as $\alpha$ increases towards the cut off. An order of magnitude estimate based in Fig. 12 would suggest that somewhere in the region of ten or possibly twenty objects of $18.0<B_{\mathrm{J}_{25}}<19.0$ might have been excluded by the limit in $\alpha$ alone.

As for stellar contamination, this is unlikely to be a serious problem as objects for which $\alpha$ exceeded -0.065 were excluded. Only in a couple of cases was there any uncertainty as to whether an object was a star or a galaxy, as all galaxy candidates were visually inspected. Overlapping stellar images were occasionally difficult to distinguish from elliptical galaxies, but it is extremely unlikely that stellar contamination could account for even $0.25 \%$ of the objects catalogued (i.e. about three objects in total).

From Fig. 13 it is evident that the VPC galaxy sample is complete to $B_{\mathrm{J}_{25}}=18.5$ for Fields $\mathrm{A}$ and $\mathrm{B}$ (assuming $\left.B_{\mathrm{J}_{25}}(\mathrm{~J} 4882)-B_{\mathrm{J}_{25}}(\mathrm{~J} 9229) \leq 1.0\right)$. It should be noted that the slight asymmetry in the distribution of data points about $B_{\mathrm{J}_{25}}(\mathrm{~J} 4882)-B_{\mathrm{J}_{25}}(\mathrm{~J} 9229)=0.0$ is due to the sensitivity of J4882 being less consistent than that of J9229. As a consequence, the most wayward data-points tend to correspond to images that yielded higher magnitudes on J4882 than on J9229. For Fields C and D, there is almost certainly completeness at $B_{\mathrm{J}_{25}}=17.5$, but galaxy loss probably only becomes significant beyond $B_{\mathrm{J}_{25}}=18.0$, as can be seen from Fig. 14 .

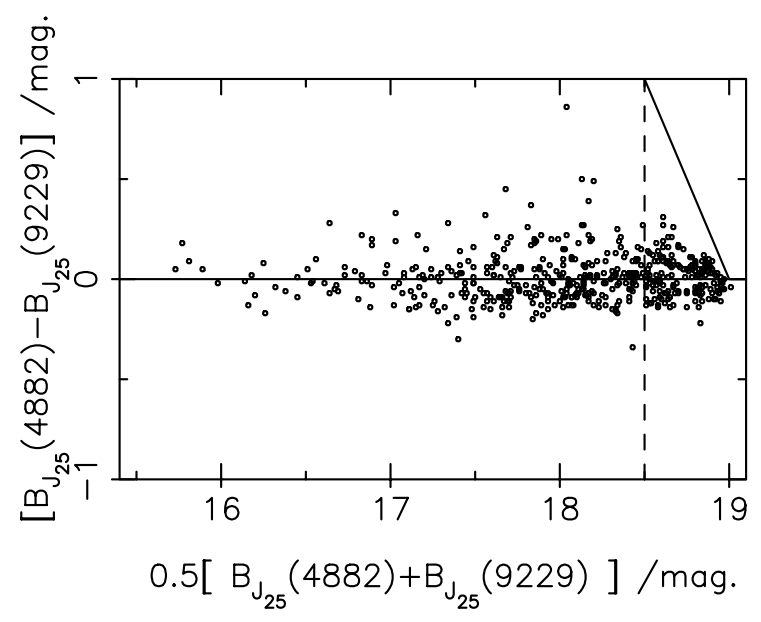

Fig. 13. The disparity between magnitudes generated from different $B_{\mathrm{J}}$ plates as a function of mean magnitude [as quoted in the VPC] for Fields A and B combined. The completeness limit (dashed line) and the observational selection criterion, $B_{\mathrm{J}_{25}}(J 4882) \leq 19.0$, (solid diagonal line) are shown for reference

\subsection{Galaxy colours}

Equal-area and total $\left(U-B_{\mathrm{J}}\right)$ and $\left(B_{\mathrm{J}}-R_{\mathrm{C}}\right)$ colours have been computed for most VPC galaxies; the main exceptions generally being those whose images were saturated in one or both relevant pass bands, and/or those that appeared heavily merged with adjacent images (whether galaxies or stars). For the equal-area colours, the areas were generally defined by the $\mu_{U}=24.25 \mathrm{mag} \operatorname{arcsec}^{-2}$ or the $\mu_{B_{\mathrm{J}}}=24.25 \mathrm{mag} \operatorname{arcsec}^{-2}$ isophote. The total colours were based on the differences between extrapolated $U_{\mathrm{t}}$, $B_{\mathrm{J}_{\mathrm{t}}}$ and $R_{\mathrm{C}_{\mathrm{t}}}$ values, and as a whole are probably less susceptible to systematic effects even though they exhibit a considerably larger scatter. However, for any individual galaxy, the equal-area colours are generally more reliable than the total ones (see Sects. 7.2 and 7.3). It was also possible to estimate very approximate total $\left(U-B_{\mathrm{J}}\right)$ colours for many of the saturated objects by means of Eq. (5) 


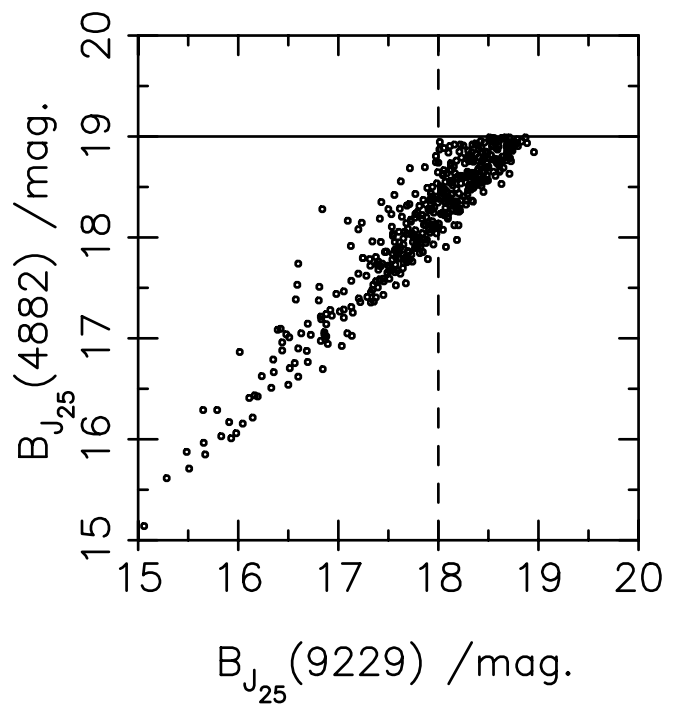

Fig. 14. The completeness limit (dashed line) to the galaxy sample in the cases of Fields C and D. Note that due to pronounced de-sensitisation of Plate J4882's emulsion over Fields $\mathrm{C}$ and $\mathrm{D}$, a mean zero-point shift of $0.31 \mathrm{mag}$ was observed between those magnitudes from Fields C and D of Plate J9229 [which were included in the VPC] and those due to J4882 for the same fields [which were discarded]

(which is an approximation based on Eq. (1)) from values of $(U-B)_{\mathrm{T}}$ and $(B-V)_{\mathrm{T}}$ whenever listed in the RC3. Unfortunately though, published $\left(B_{\mathrm{J}}-R_{\mathrm{C}}\right)$ colours could not be found for those objects whose images were saturated.

$\left(U-B_{\mathrm{J}}\right)_{\mathrm{t}} \approx(U-B)_{\mathrm{T}}+0.35(B-V)_{\mathrm{T}}$.

\subsection{Radial velocities}

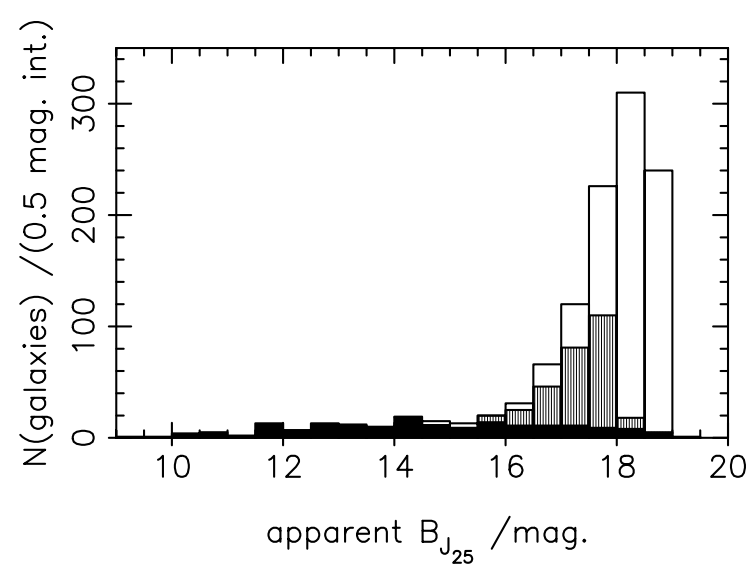

Fig. 15. Frequency distribution of VPC galaxies still lacking published velocities (unshaded areas), those measured for the first time by Drinkwater et al. (1996) (vertically shaded areas) and those measured previously (unshaded); all as a function of $B_{\mathrm{J}_{25}}$
Only a minority of galaxies fainter than $B_{\mathrm{J}_{25}}=17.50$ have published radial velocities, but the situation has improved since Drinkwater et al.'s (1996) study. The present situation is depicted in Fig. 15.

\subsection{Morphological types}

Morphological types are essentially complete to $B_{\mathrm{J}_{25}}=$ 17.00, but sporadic beyond this limit as shown by Fig. 16 . As the Du Pont plates of Binggeli et al. (1985) had considerably larger plate scales than did our UKST plates, VCC types have been adopted for almost all of the galaxies in common between the VPC and the VCC galaxy samples. Those galaxies which were brighter than $B_{\mathrm{J}_{25}}=17.00$ yet not included in the VCC (generally because they were deemed to be background objects) were classified by visual inspection of a copy plate of UKST plate J9229. Due to the small scale of this copy plate and the problem of image saturation in many cases, no attempt has been made to provide detailed morphological classifications (e.g. spiral subclasses or luminosity classes) in the VPC.

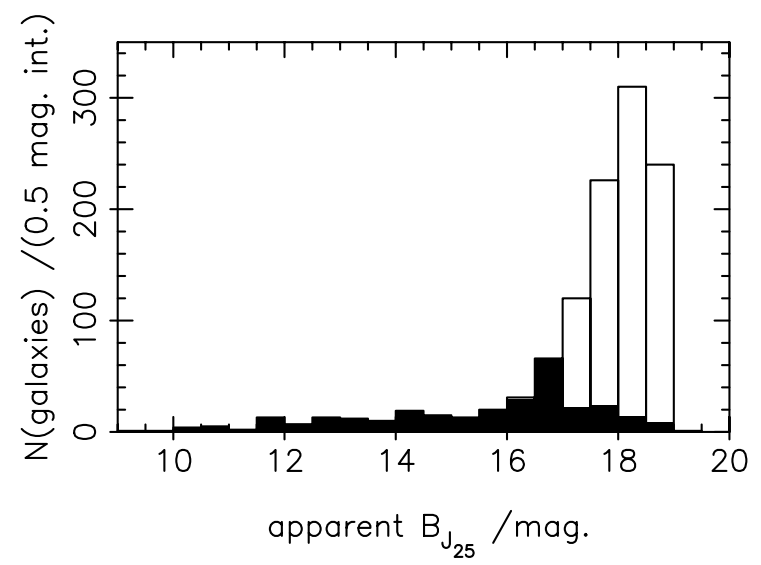

Fig. 16. Frequency distribution of VPC galaxies that have been typed (heavily shaded areas) and untyped (unshaded areas) as a function of $B_{\mathrm{J}_{25}}$. Note that any unshaded area brightward of $B_{\mathrm{J}_{25}}=17.00$ represents galaxies which defied typing

\subsection{Astrometry}

Unambiguous identification of the vast majority of galaxies within the central Virgo field (background objects included) brighter than $B_{\mathrm{J}_{25}} \sim 18.5$ should now be possible, as the use of a measuring machine has enabled improved positions to be computed. Only for a subset of objects saturated in $B_{\mathrm{J}}$, was it necessary to extract the coordinates from the RC3 or occasionally from the VCC, though these objects are generally so extended that the positional accuracy required to identify them unambiguously is not that high. The VPC also contains orientation and ellipticity information. 


\section{Sources of error}

\subsection{The photometry (general)}

The issue of saturation effects due to limitations in the dynamic range of the COSMOS measuring machine has already been dealt with at length in Sect. 3.5. The other most common causes of systematic errors in photographic photometry include: differential de-sensitisation of photographic emulsions due to exposure to atmospheric oxygen and water-vapour, vignetting, uneven and/or inadequate hypersensitisation (these effects can prevent the sky density reaching the threshold necessary for a linear response on those areas of the emulsion affected) plate defects (such as scratches or spurious "images" that are wholly artefact) and inadequacies in background-fitting, sky-subtraction and calibration procedures (which also depend on the accuracy of the reference measurements e.g. photoelectric aperture- [or simulated-aperture-] photometry measurements).

As can be seen from Fig. 13 and Fig. 14, for Fields $\mathrm{A}$ and $\mathrm{B}$ the mean zero-point offset between the isophotal magnitudes generated from the different $B_{\mathrm{J}}$ plates is small: $B_{\mathrm{J}_{25}}(J 4882)-B_{\mathrm{J}_{25}}(J 9229)=+0.014$, whilst for Fields $\mathrm{C}$ and $\mathrm{D}$ it is quite substantial: $B_{\mathrm{J}_{25}}(J 4882)-$ $B_{\mathrm{J}_{25}}(J 9229)=+0.311$. Significant de-sensitisation of Plate J4882 over most of Fields C and D was found to be the cause of this disparity. J4882 was the inferior plate of the two, almost certainly because it was taken before an important modification was made to the UKST plateholder in December 1982. This modification enabled the plate holder to be flushed with dry nitrogen (which is inert as far as the emulsions are concerned) during exposures, thus preventing de-sensitisation. Note that as J4882 is quite a dark plate (i.e. emulsion densities due to the sky are quite high) the problem in this case was not that the differential de-sensitisation depressed the sky brightness below the threshold for a linear response on the part of the emulsion, but rather that the relative density-tointensity calibration was based largely on that part of the emulsion's characteristic-response curve between the linear portion and the saturated portion. As the sky was very dense throughout Fields $\mathrm{A}$ and $\mathrm{B}$, this calibration was quite adequate for these fields. However, over large areas of Fields C and D, the sky density was depressed onto the central part of the characteristic curve's linear portion for which the adopted relative-calibration polynomial was poorly determined.

As the $B_{\mathrm{J}}$ magnitudes were based on both plates in the cases of Fields A and B, but by necessity just on Plate J9229 in the cases of Fields C and $\mathrm{D}^{4}$, the errors are $\sqrt{2}$ higher for the latter fields. Mean standard errors on those

\footnotetext{
4 Note that strictly speaking, due to the slight displacement between the J9229 and J4882 $(x, y)$ coordinate systems, those images near to the boundaries of any field in one system may lie within a neighbouring field in the other system. In these cases and when a plate flaw rendered one plate unreliable for
}

mean magnitudes that were derived from two plates are tabulated in Table 6 , and were multiplied by $\sqrt{2}$ in order to obtain values applicable to magnitudes derived from one plate. Corresponding errors on the reduced $\mu_{B_{\mathrm{J}}}=$ $25.00 \mathrm{mag} \operatorname{arcsec}^{-2}$ isophotal radii are shown in Table $7^{5}$.

Table 6. Mean standard errors on the $B_{\mathrm{J}_{25}}$ magnitudes quoted in the VPC as a function of the number of plates on which the measurements were based, and as a function of mean apparent $B_{\mathrm{J}_{25}}$

\begin{tabular}{lcc}
\hline magnitude range & two plates & one plate \\
\hline $15.5 \leq B_{\mathrm{J}_{25}}<16.5$ & 0.06 & 0.08 \\
$16.5 \leq B_{\mathrm{J}_{25}}<17.5$ & 0.08 & 0.11 \\
$17.5 \leq B_{\mathrm{J}_{25}}<18.5$ & 0.10 & 0.14 \\
\hline
\end{tabular}

Table 7. Mean standard errors on the reduced $\mu_{B_{\mathrm{J}}}=25.00$ mag $\operatorname{arcsec}^{-2}$ isophotal radii in the VPC, expressed as percentages of the corresponding radii measurements, and shown as functions of both the number of plates on which the measurements were based and apparent $B_{\mathrm{J}_{25}}$

\begin{tabular}{lcc}
\hline magnitude range & two plates & one plate \\
\hline $15.5 \leq B_{\mathrm{J}_{25}}<16.5$ & $4.9 \%$ & $6.9 \%$ \\
$16.5 \leq B_{\mathrm{J}_{25}}<17.5$ & $4.5 \%$ & $6.4 \%$ \\
$17.5 \leq B_{\mathrm{J}_{25}}<18.5$ & $4.0 \%$ & $5.7 \%$ \\
\hline
\end{tabular}

Vignetting arises as a consequence of geometric shadowing effects inherent to many telescope designs including that of the UKST. The net effect is that off-axis light is not transmitted as efficiently to the image plane as is on-axis light, with the fractional reduction in efficiency increasing with off-axis distance. In the case of the UKST, the fractional reduction in efficiency is $<1 \%$ within $\sim 2.7$ of the axis. As the survey area is 4.8 across and more or less centred on the plate centre, only four small areas near to the corners of the survey area could possibly be affected, if the sky density failed to reach the threshold for a linear response (on the part of the photographic emulsion) within these regions. In Sect. 3.3, it was mentioned that

a particular object, whether a quoted magnitude was based on one or two plates depended on other factors. Individual cases can be checked in the VPC which quotes standard errors on the machine-generated (mean) $B_{\mathrm{J}_{25}}$ magnitudes only when two plates were used.

5 As with the mean standard errors on the colours quoted in the VPC, the lower values obtained for fainter objects may be due to limitations in the dynamic range of the COSMOS microdensitometer. 
Table 8. Quantification of the effect of the 5 -arcsec FWHM resolution function on model galaxy profiles ${ }^{\text {a }}$ for a variety of Sérsic parameters $\left(n\right.$ and $\left.\mu_{B_{\mathrm{J}}}(0)\right)$ and isophotal radii $r_{B_{\mathrm{J}_{25}}}$

\begin{tabular}{|c|c|c|c|c|c|}
\hline$n$ & $\mu(0)$ & $r_{B_{\mathrm{J}_{25}}}=4^{\prime \prime}$ & $r_{B_{\mathrm{J}_{25}}}=6^{\prime \prime}$ & $r_{B_{\mathrm{J}_{25}}}=8^{\prime \prime}$ & $r_{B_{\mathrm{J}_{25}}}=10^{\prime \prime c}$ \\
\hline 0.25 & 16.5 & $+13+0.16>17.7$ & $+7+0.06>17.4$ & $+5+0.03>17.2$ & $+3+0.01>16.9$ \\
\hline 0.50 & 22.0 & {$[+3+0.0619 .7]^{\mathrm{b}}$} & $+3+0.0419 .0$ & $+3+0.0318 .4$ & $-1-0.0217 .9$ \\
\hline 0.50 & 23.5 & {$\left[\begin{array}{lll}-1 & -0.08 & 20.3\end{array}\right]^{\mathrm{b}}$} & {$[+1+0.0019 .4]^{\mathrm{b}}$} & $+1+0.0018 .8$ & $-5-0.1118 .4$ \\
\hline 1.00 & 22.0 & {$[+5+0.0619 .2]^{\mathrm{b}}$} & $+5+0.0418 .5$ & $+5+0.0317 .9$ & $+3+0.0117 .4$ \\
\hline 1.00 & 23.5 & {$\left[\begin{array}{lll}-1 & -0.07 & 20.1\end{array}\right]^{\mathrm{b}}$} & {$[+1+0.0019 .2]^{\mathrm{b}}$} & $+1+0.0018 .6$ & $-3-0.0618 .2$ \\
\hline 2.00 & 22.0 & $+9+0.0318 .8$ & $+9+0.0317 .9$ & $+7+0.0217 .3$ & $+5+0.0116 .9$ \\
\hline 2.00 & 23.5 & {$[+1+0.0019 .7]^{\mathrm{b}}$} & $+1+0.0018 .9$ & $+1+0.0018 .3$ & $+1+0.0017 .8$ \\
\hline
\end{tabular}

a The three entries per convolution are [left to right]:

(1) $\Delta r_{B_{\mathrm{J}_{25}}}=r_{B_{\mathrm{J}_{25}}}$ (smoothed) $-r_{B_{\mathrm{J}_{25}}}$ (unsmoothed) in units of $\frac{1}{8}{ }^{\prime \prime}$ (only correct to $\left.\pm \frac{1}{4}{ }^{\prime \prime}\right)$;

(2) $\Delta B_{\mathrm{J}_{25}}=B_{\mathrm{J}_{25}}$ (smoothed) $-B_{\mathrm{J}_{25}}$ (unsmoothed); and

(3) approximate $B_{\mathrm{J}_{25}}$ (smoothed)

b Entries corresponding to objects too faint to be included in the VPC are enclosed in square brackets

c For images with $r_{B_{\mathrm{J}_{25}}}>10^{\prime \prime}$, the percentage changes will of course be smaller still.

the skies were established to be flat. In order to test for the significance of any apparent slope in the estimated sky as a function of radial distance from a plate's centre, the sky values estimated for the positions of those galaxies with published aperture photometry measurements were plotted as a function of radial distance.

Although, in the case of the U9362 (see Fig. 17) there does at first sight appear to be a slight fall off in estimated sky brightness as a function of off-axis distance, the size of the slope was found to be $-0.017 \pm$ $1.08 \mathrm{mag} \operatorname{arcsec}^{-2} \mathrm{deg}^{-1}$. In other words, the uncertainty on the slope is very much larger than the best estimate of the slope. In the case of J9229, there is even less evidence for any radial variation as this slope was found to be $-0.008 \pm 1.41 \mathrm{mag} \operatorname{arcsec}^{-2} \mathrm{deg}^{-1}$. These slopes were computed by means of bootstrap resampling with 10000 realisations in each case, and using as many data points for each galaxy as there were individual aperture measurements for that galaxy; even though only one data point (the mean sky estimate) is shown in Fig. 17 for each galaxy's position. In summary then, whilst more calibrating galaxies beyond the 2.7 limit would really be required to establish conclusively that vignetting had been properly accounted for, no evidence has been found for significant radial variation in the skies of Plates J9229 and U9362. This does not of course rule out the possibility that there is significant radial variation, but it does establish that the random disagreements between other observers' aperture photometry measurements constitute a much larger effect.

The $1 \sigma$ ranges of sky density values for Plate J9229 in Fields A, B, C and D were $635-679,660-690,540-700$ and 545-690 density units respectively. Ignoring localised regions affected by prominent objects, the sky density was subject to variation of the order of $10 \%$ (625 - 690 units) over $95.8 \%$ of the scanned area of Plate J9229. The remaining $4.2 \%$ corresponded to the corners of the combined area of the four fields, where vignetting reduced the densities. The vignetting was most severe in the outer corners of Fields C and D, where the densities were reduced to about 550 units. Apart from the issue of how well vignetting has been compensated for (which has already been dealt with) the accuracy to which the sky could be determined depended on the noise associated with the background measurements and any bias there may have been in the determination of the mode sky density values to which the background fits were applied.

The bias in the estimation of mode densities was found to be of the order of $0.06 \pm 0.02 \%$ of a sky unit, in the sense that the sky brightness was slightly overestimated. This figure of $0.06 \%$ was arrived at by comparing the adopted estimate of the mode for each bin (containing 7225 pixels) with that mode value obtained by interpolating a histogram of density values for that bin. As described in Sect. 3.2, there were typically $2000+$ bins not significantly affected by extended objects. The interpolation process involved fitting a Gaussian curve to the central portion of each histogram's modal peak, using Starlink's ESP package.

The sample standard deviation in the sky values with respect to the background fits was less than $0.25 \%$ in sky units $(0.20 \%, 0.22 \%, 0.23 \%$ and $0.25 \%$ for Fields A, B, C and $\mathrm{D}$ respectively). This is considerably larger than the bias effect which will henceforth be neglected. The VPC's limiting $B_{\mathrm{J}}$-isophote of $25.0 \mathrm{mag} \operatorname{arcsec}^{-2}$, was $5.6 \%$ of Plate J9229's sky intensity. The random component of the 
uncertainty on the limiting isophote due to background fitting errors is therefore at most $\pm 0.05 \mathrm{mag} \operatorname{arcsec}^{-2}$ for Plate J9229.

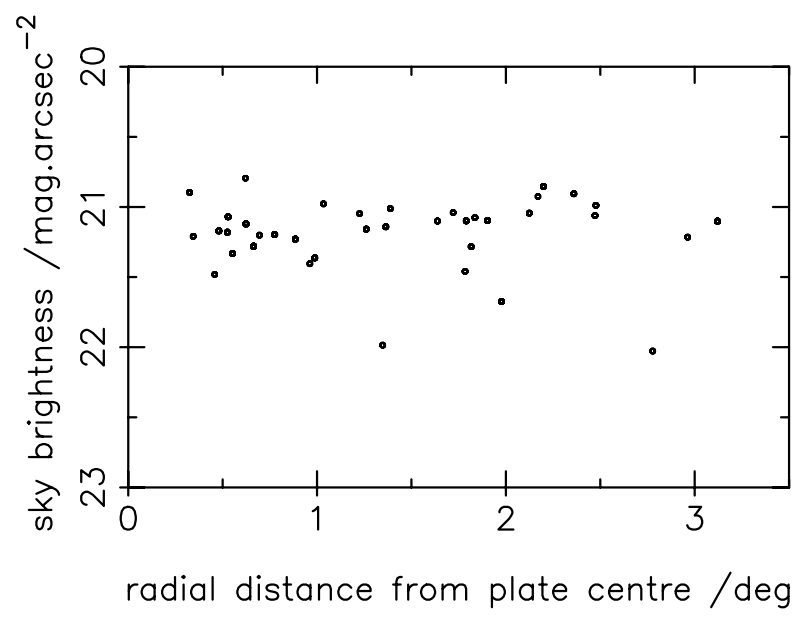

Fig. 17. The estimated surface brightness of the sky on Plate U9362 for the positions of galaxies with published $U$-band aperture photometry, as a function of radial distance from the plate centre. Note that the points plotted are means for each galaxy, and that some points are very much more significant than others due to either (1) being based on more aperture photometry measurements and/or (2) because they are based on more independent observers' measurements

One of the advantages of having a slight displacement between the different J9229 and J4882 fields was that if an image happened to be truncated by the edge of a field in one coordinate system, it was unlikely to be truncated in the other system. It was therefore possible to base the $B_{\mathrm{J}}$ magnitudes of such galaxy images on the most suitable plate. As a result, edge-of-field effects have been minimised, though Fig. 18 was plotted in order to provide a check on the effectiveness of the background-fitting procedure at the extremities of a field where interpolation was not possible. As can be seen from this figure, the magnitude measurements do tend to be noisier at the field edges than at the field centres, but the increase in noise with distance from the field centres does appear to be quite gradual and without any very steep increase near the edges.

Overlaps between adjacent images could not always be resolved satisfactorily during the image segmentation. Magnitudes and indeed other photometric parameters are therefore less reliable for images with overlapping isophotes, and particularly so in cases where the brightest overlapping isophote approaches the mean (or even the peak) surface brightness quoted for the galaxy.

As the measured mean and peak surface-brightness parameters (as opposed to the extrapolated ones) quoted in the VPC have not been corrected for effects that degrade image resolution; namely diffraction, atmospheric

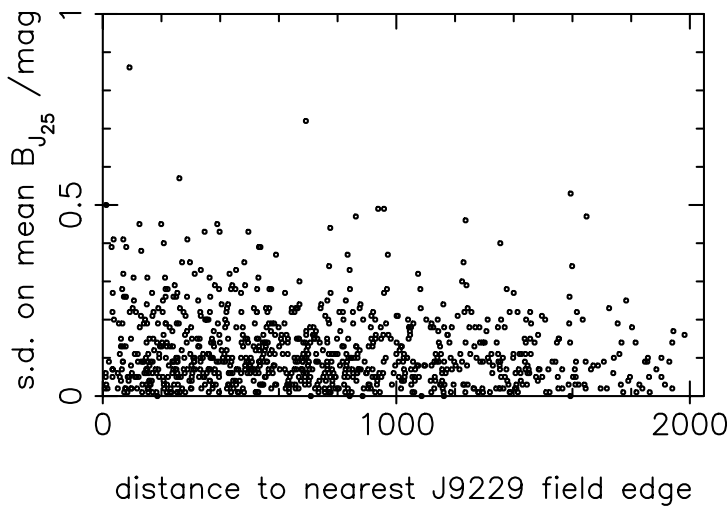

Fig. 18. Individual standard errors on those mean $B_{\mathrm{J}}$ magnitudes that were derived from both $B_{\mathrm{J}}$ plates (overlapping images excluded) as a function of distance from the nearest field edge measured in 2. . 1 pixels

seeing, sampling due to the pixel size of the plate scans and the smoothing of the scans prior to image segmentation; they tend to be slight over-estimates of the true values as measured in mag $\operatorname{arcsec}^{-2}$. Unsaturated compositestellar-profiles (as described in Sect. 3.5) were measured in order to estimate the degree to which the resolution of each image had been degraded by smearing effects, particularly the smoothing of the plate-scan data. The FWHM of the unsaturated composite stellar profiles were found to be $44^{\prime \prime} 75 \pm 0$.' 1 in the case of U9362, $5^{\prime \prime} \pm 00^{\prime \prime} 1$ in the cases of the two $B_{\mathrm{J}}$ plates and $6^{\prime \prime} \pm 0^{\prime \prime} .1$ in the case of R2936.

Of the four plates reduced, R2936 (for which the limiting isophote adopted corresponded to $10.4 \%$ of the sky) was the noisiest, followed by J4882 (7.7\%), J9229 (5.7\%) and the least noisy U9362 (3.1\%). It was unfortunate that without such severe smoothing, limiting isophotes $0.5 \mathrm{mag} \operatorname{arcsec}^{-2}$ brighter would probably have to have been adopted as noise peaks in the plate-scan data would have degraded the images around their edges. The smoothing was also very necessary to reduce image fragmentation (the segmentation of individual galaxy images into two or more major components).

In order to investigate the extent to which the VPC's $B_{\mathrm{J}}$ isophotal magnitudes have been degraded by atmospheric seeing, sampling and smoothing; modelgalaxy profiles were convolved with a $5^{\prime \prime}$ FWHM Gaussian $^{6}$ distribution. Only relatively small images were considered, as the significance of the degradation decreases with increasing image size. The results are tabulated in Table 8 , in which the approximate changes in isophotal radius, $r_{B_{\mathrm{J}_{25}}}$, and isophotal magnitude, $B_{\mathrm{J}_{25}}$, are shown for a variety of different Sérsic profile parameters (intrinsic index, $n$, and intrinsic central surface brightness, $\left.\mu_{B_{\mathrm{J}}}(0),\right)$ and for a variety of small intrinsic isophotal radii, $r_{B_{J_{25}}}$.

\footnotetext{
6 To be rigorous, the resolution function is not of course a perfect Gaussian (or identical for different parts of any plate) but a Gaussian is still a good approximation.
} 
As can be seen from Table 8, even though the effects on the isophotal radii can be quite significant, the errors introduced to the isophotal magnitudes are typically of the order of several percent (i.e. several hundredths of a magnitude) even for the small images considered, and are in most cases insignificant compared to the other sources of error quantified earlier. Also, note that a coarse resolution function can cause observed isophotal radii to be $e i$ ther larger or smaller than their intrinsic values, in other words the effect is not uni-directional.

\subsection{Equal-area colours}

The standard error on each mean colour, as quoted in the VPC, is based upon two colour values generated from either of the two following plate combinations:

1) U9362 and J4882, U9362 and J9229

2) R2936 and J4882, R2936 and J9229.

Assuming that U9362 and R2936 are of similar quality to the $B_{\mathrm{J}}$ plates, the standard errors quoted in the VPC need to be multiplied by $\sqrt{2}$ in order to obtain meaningful estimates of the standard errors on the quoted colours, as tabulated in Table 9 .

Table 9. Estimated mean standard errors on colours in the VPC, as a function of the number of plates on which the measurements were based, and as a function of mean apparent $B_{\mathrm{J}_{25}}$

\begin{tabular}{lcccc}
\hline magnitude range & \multicolumn{2}{c}{$\left(U-B_{\mathrm{J}}\right)$} & \multicolumn{2}{c}{$\left(B_{\mathrm{J}}-R_{\mathrm{C}}\right)$} \\
& 2 plates & 1 plate & 2 plates & 1 plate \\
\hline $15.5 \leq B_{\mathrm{J}_{25}}<16.5$ & 0.18 & 0.20 & 0.19 & 0.22 \\
$16.5 \leq B_{\mathrm{J}_{25}}<17.5$ & 0.17 & 0.19 & 0.16 & 0.18 \\
$17.5 \leq B_{\mathrm{J}_{25}}<18.5$ & 0.16 & 0.18 & 0.16 & 0.18 \\
\hline
\end{tabular}

Systematic errors must also be present due to differences between the resolution functions inherent to different plate scans. Systematic differences between the equalarea and total colours were however found to be very small in the case of the $B_{\mathrm{J}}-R_{\mathrm{C}}$ index, though more noticeable in the case of the $U-B_{\mathrm{J}}$.

\subsection{Total magnitudes and colours}

The estimated random errors on the total magnitudes quoted in the VPC are listed in Table 10. They are based on several assumptions and approximations, and are therefore only intended as rough estimates. The mean extrapolation from $B_{\mathrm{J}_{25}}$ to $B_{\mathrm{J}_{\mathrm{t}}}$ is $0.35 \mathrm{mag}$, and the estimated standard error on a mean extrapolation was taken to be $0.35 \div 3$ magnitudes. This value was propagated with each of the mean standard errors on $B_{\mathrm{J}_{25}}$ values listed in Table(s) 6 (and 10) to yield mean standard-error estimates on $B_{\mathrm{J}_{\mathrm{t}}}$ values. From Fig. 8 it can be seen that the uncertainty on any estimate of $(B-V)$ based on a value of
$\left(B_{\mathrm{J}}-R_{\mathrm{C}}\right)$ is $\sim 0.2 \mathrm{mag}$. The offset between the $B$ and $B_{\mathrm{J}}$ systems is $\sim 0.35(B-V)$, whence the mean error in the $B_{\mathrm{J}_{\mathrm{t}}}$-to- $B_{\mathrm{t}}$ conversion was taken to be 0.07 magnitudes. This was increased to 0.1 magnitude in order to take into account uncertainties in the colour-coefficient term, and propagated with the mean standard deviation estimates for $B_{\mathrm{J}_{\mathrm{t}}}$ values in order to obtain mean standard deviation estimates on $B_{\mathrm{t}}$ values. The mean extrapolations from $U_{25}$ to $U_{\mathrm{t}}$ and from $R_{\mathrm{C}_{24}}$ to $R_{\mathrm{C}_{\mathrm{t}}}$ were found to be 0.45 and $0.25 \mathrm{mag}$ respectively. Therefore the random errors on the $\left(B_{\mathrm{J}}-R_{\mathrm{C}}\right)_{\mathrm{t}}$ colours can be expected to be considerably smaller than those on the $\left(U-B_{\mathrm{J}}\right)_{\mathrm{t}}$ ones. This was confirmed by intercomparisons between the equal-area and total colours.

Table 10. Estimated mean standard errors on the isophotal and total magnitudes quoted in the VPC, as a function of the number of plates on which the measurements were based, and as a function of mean apparent $B_{\mathrm{J}_{25}}$

\begin{tabular}{lccc}
\hline Magnitude range & $\sigma\left(B_{\mathrm{J}_{25}}\right)$ & $\sigma\left(B_{\mathrm{J}_{\mathrm{t}}}\right)$ & $\sigma\left(B_{\mathrm{t}}\right)$ \\
\hline Two plates: & & & \\
$15.5 \leq B_{\mathrm{J}_{25}}<16.5$ & 0.06 & 0.13 & 0.16 \\
$16.5 \leq B_{\mathrm{J}_{25}}<17.5$ & 0.08 & 0.14 & 0.17 \\
$17.5 \leq B_{\mathrm{J}_{25}}<18.5$ & 0.10 & 0.15 & 0.18 \\
$\quad$ One plate: & & & \\
$15.5 \leq B_{\mathrm{J}_{25}}<16.5$ & 0.08 & 0.14 & 0.17 \\
$16.5 \leq B_{\mathrm{J}_{25}}<17.5$ & 0.11 & 0.16 & 0.19 \\
$17.5 \leq B_{\mathrm{J}_{25}}<18.5$ & 0.14 & 0.18 & 0.21 \\
\hline
\end{tabular}

\subsection{Galaxy magnitudes from other sources}

In the absence of alternative measurements for most of the objects suffering from saturation effects in the VPC, those sources listed in Sect. 5 have had to suffice. It is hoped that for the bright galaxies concerned, these sources are reasonably reliable.

\subsection{Equatorial coordinates}

An indication as to the accuracy of the astrometry is given by the residuals between the positions as quoted in the AGK3 catalogue and those calculated according to the plate solution adopted. The root-mean-squared residual was 2 .'28 (0." 87 in right ascension and 2 "' 11 in declination). As the segmentation software did not centroid the galaxy images, but assigned $(x, y)$ coordinates to the brightest pixel within each image, this quantity is, as expected, quite similar to the pixel size of the segmented images: 2 "'148. The lack of a centroiding algorithm was not a problem for the saturated galaxies however, as RC3 positions were generally adopted for them. 


\subsection{Radial velocities}

These errors were taken from those sources of radialvelocities listed in Sect. 5 without modification. In the cases of the following VPC (VCC) objects, there are very large mutual disagreements between different sources in the literature, and the true errors may be very much larger than the values quoted: VPC 25, 424, 447, 502, 545, 624 and 810 (which correspond to VCC 325, 815, 870, 945, 1035,1148 and 1355 respectively).

\subsection{Morphological types}

Owing to the small plate scale of the UKST plates, and the high degree of saturation exhibited by many of the galaxy images, even approximate typing was not always possible. The majority of those morphological types obtained from the VCC (see Sect. 5) were presumed to be correct, but one mis-classification was noticed. Binggeli et al.'s (1985) VCC 500 was classified as an S0, instead of as a ringed-spiral. This has been corrected in the VPC. Note that the re-classifications listed in de Vaucouleurs \& Corwin (1986) all concern spiral-galaxy subclasses, and are therefore beyond the scope of the broad classifications provided by the VPC.

\section{Comparisons with photoelectric photometry}

\subsection{Aperture photometry}

Photographic radial integrated-light profiles were generated for all unsaturated galaxy images for which comparable photoelectric aperture and/or simulated-aperture photometry measurements could be found in the literature. These integrated-light profiles were then interpolated to radii corresponding to the apertures used by other observers yielding Tables 11, 12 and 13. The integrations were performed using unsmoothed plate-scan data. Other observers' $B$ - and $V$-band measurements were transformed into the $B_{\mathrm{J}}$ system by means of Eq. (1). Note that no comparisons have been made with published measurements made with apertures of radii less than 12 arcsec. This is because, at small apertures, comparisons are particularly sensitive to differences in the angular resolutions of the images concerned.

Four unsaturated VPC objects have been measured by observers at McDonald Observatory of the University of Texas (de Vaucouleurs 1959; de Vaucouleurs et al. 1978; de Vaucouleurs et al. 1981) as listed in Table 11. In the blue, de Vaucouleurs et al. find IC $3481 \mathrm{~A}$ to be $\sim 1.7$ mag brighter than we do. Interestingly, in the VPC, the adjacent galaxy, IC 3481, is also found to be $\sim 1.7 \mathrm{mag}$ brighter than IC 3481A. Assigning equal weights to each galaxy, the apparent mean zero-point offsets were found to be $\Delta U=U$ (Texas) $-U(\mathrm{VPC})=-0.33 \mathrm{mag}$ with a sample standard deviation of $\sigma_{N-1}=0.76 \mathrm{mag}$ and $\Delta B_{\mathrm{J}}=B_{\mathrm{J}}($ Texas $)-B_{\mathrm{J}}(\mathrm{VPC})=-0.48\left(\sigma_{N-1}=0.87\right)$
Table 11. Galaxies in common with the VPC, that have been measured by observers at McDonald Observatory of the University of Texas ${ }^{\mathrm{a}}$

\begin{tabular}{lrccccc}
\hline $\mathrm{IC}$ & $r /^{\prime \prime}$ & $B_{\mathrm{J}_{9229}}$ & $B_{\mathrm{J}_{4882}}$ & $U_{9362}$ & $\Delta B_{\mathrm{J}}^{\mathrm{b}}$ & $\Delta U^{\mathrm{c}}$ \\
\hline 3355 & 32.9 & 15.89 & 15.86 & 15.83 & -0.40 & -0.43 \\
3355 & 65.6 & 15.49 & 15.82 & 15.53 & -0.29 & -0.33 \\
3475 & 53.3 & 14.57 & - & 15.12 & -0.05 & -0.21 \\
3475 & 65.6 & 14.38 & - & 14.94 & -0.09 & -0.30 \\
3475 & 68.7 & 14.34 & - & 14.90 & -0.09 & -0.32 \\
3475 & 104.0 & 14.07 & - & - & +0.00 & - \\
$3481 \mathrm{~A}$ & 41.4 & 15.85 & - & 16.37 & -1.73 & -1.26 \\
3483 & 24.4 & 15.26 & - & 15.45 & +0.25 & +0.61 \\
\hline
\end{tabular}

a Their measurements are compared with $U$ and $B_{\mathrm{J}}$ magnitudes integrated radially to radii corresponding to their apertures

${ }^{\mathrm{b}} \Delta B_{\mathrm{J}}=0.65 B($ Texas $)+0.35 V($ Texas $)-B_{\mathrm{J}}(\mathrm{VPC})$

c $\Delta U=U($ Texas $)-U(\mathrm{VPC})$.

Table 12. Objects in common between the VPC and Gallagher \& Hunter $(1986)^{a}$

\begin{tabular}{lcccccc}
\hline $\mathrm{IC} / \mathrm{VCC}$ & $r /^{\prime \prime} b^{b}$ & $B_{\mathrm{J}_{9229}}$ & $B_{\mathrm{J}_{4882}}$ & $U_{9362}$ & $\Delta B_{\mathrm{J}}{ }^{\mathrm{c}}$ & $\Delta U^{\mathrm{d}}$ \\
\hline $\mathrm{V} 1377$ & 20.0 & 16.81 & - & - & +0.41 & - \\
$\mathrm{V} 512$ & 39.5 & 16.08 & - & - & -0.20 & - \\
$\mathrm{I} 3239$ & 20.0 & 16.53 & - & - & -0.32 & - \\
$\mathrm{I} 3355$ & 30.0 & 15.97 & 15.90 & 15.92 & -0.53 & -0.63 \\
$\mathrm{I} 3416$ & 20.0 & 15.91 & - & 15.75 & +0.02 & -0.16 \\
$\mathrm{I} 3475$ & 15.0 & 16.53 & - & 16.89 & -0.15 & -0.04 \\
\hline
\end{tabular}

a Their measurements are compared with $U$ and $B_{\mathrm{J}}$ magnitudes integrated radially to radii corresponding to their apertures

b Measurements corresponding to apertures of radius $<12^{\prime \prime}$ have not been included because they can be expected to be particularly sensitive to differences in seeing conditions

c $\Delta B_{\mathrm{J}}=0.65 B(\mathrm{G} \& \mathrm{H})+0.35 V(\mathrm{G} \& \mathrm{H})-B_{\mathrm{J}}(\mathrm{VPC})$

${ }^{\mathrm{d}} \Delta U=U(\mathrm{G \& H})-U(\mathrm{VPC})$.

mag. Removing IC $3481 \mathrm{~A}$ from the sample, reduces the residuals to $\Delta U=-0.02\left(\sigma_{N-1}=0.55\right) \mathrm{mag}$ and $\Delta B_{\mathrm{J}}=$ $-0.06\left(\sigma_{N-1}=0.30\right) \mathrm{mag}$. As there is a tendency for the residuals to be smaller, the larger the aperture employed, it is quite possible that resolution differences are responsible for many of the apparent discrepancies.

Gallagher \& Hunter (1986) measured six galaxies with sufficiently large apertures for comparisons to be useful. These objects are listed in Table 12. The apparent mean zero-point discrepancies were found to be $\Delta U=$ $U(\mathrm{G} \& \mathrm{H})-U(\mathrm{VPC})=-0.14\left(\sigma_{N-1}=0.32\right) \mathrm{mag}$ and $\Delta B_{\mathrm{J}}=B_{\mathrm{J}}(\mathrm{G} \& \mathrm{H})-B_{\mathrm{J}}(\mathrm{VPC})=-0.17\left(\sigma_{N-1}=0.41\right)$ mag. However, as Gallagher \& Hunter's aperture sizes are generally quite small, a large part of the apparent discrepancies may well be due to resolution differences. In the 
Table 13. Caldwell (1983) dwarfs in common with the VPC ${ }^{a}$

\begin{tabular}{lcccccc}
\hline $\mathrm{dw} / \mathrm{VPC}$ & $r /^{\prime \prime}$ & $B_{\mathrm{J}_{9229}}$ & $B_{\mathrm{J}_{4882}}$ & $U_{9362}$ & $\Delta B_{\mathrm{J}}^{\mathrm{b}}$ & $\Delta U^{\mathrm{c}}$ \\
\hline $1 / 843$ & 17.8 & 15.87 & - & 16.15 & -0.42 & -0.14 \\
$3 / 856$ & 17.8 & 16.86 & - & 17.54 & -0.20 & -0.37 \\
$6 / 801$ & 19.5 & 15.99 & - & 16.37 & -0.14 & +0.12 \\
$8 / 835$ & 17.8 & 16.60 & - & 16.92 & -0.28 & -0.09 \\
$11 / 834$ & 17.8 & 16.04 & - & 16.42 & -0.29 & -0.19 \\
$12 / 808$ & 19.5 & 17.43 & - & 17.91 & +0.00 & -0.11 \\
$27 / 420$ & 17.8 & 17.06 & 17.31 & 17.50 & -0.29 & -0.01 \\
$28 / 342$ & 17.8 & 16.10 & 16.07 & 16.27 & -0.34 & -0.11 \\
$31 / 937$ & 17.8 & 16.57 & - & 16.92 & -0.09 & +0.00 \\
\hline
\end{tabular}

a Caldwell's measurements are compared with $U$ and $B_{\mathrm{J}}$ magnitudes integrated radially to radii corresponding to his apertures

b $\Delta B_{\mathrm{J}}=0.65 B($ Caldwell $)+0.35 V($ Caldwell $)-B_{\mathrm{J}}(\mathrm{VPC})$

c $\Delta U=U($ Caldwell $)-U(\mathrm{VPC})$.

case of the most discrepant galaxy, IC 3355 (which was also measured by de Vaucouleurs et al.), there may be an additional explanation. IC 3355 lies only $\sim 15$ arcmin from the direction of the giant elliptical, NGC 4406, and the prominent galaxies NGCs 4435, 4438 and 4402 are even closer at hand. As IC 3355 's direction is so central within the cluster, photometry of this galaxy may therefore be very sensitive to the background procedures adopted, due to the extended haloes of the giant galaxies in the vicinity and/or any intra-cluster light that may be present.

There are nine VPC objects in common with Caldwell's (1983) sample of dwarfs ${ }^{7}$ as listed in Table 13. The apparent mean zero-point shifts were found to be $\Delta U=U($ Caldwell $)-U(\mathrm{VPC})=-0.10\left(\sigma_{N-1}=0.14\right)$ $\operatorname{mag}$ and $\Delta B_{\mathrm{J}}=B_{\mathrm{J}}($ Caldwell $)-B_{\mathrm{J}}(\mathrm{VPC})=-0.23$ $\left(\sigma_{N-1}=0.13\right)$ mag. These comparisons may however, be even more susceptible to differences in image resolution, as Caldwell's apertures tend to be smaller than those of de Vaucouleurs et al. and Gallagher \& Hunter.

\subsection{Surface photometry}

The integrated-light profiles of IC 3475 (in $B_{\mathrm{J}}$ ) and NGC 4468 (in $U$ ) are depicted in Figs. 19 and 20 respectively. NGC 4468 has been measured by several other observers, whose measurements tend to be about $0.2 \mathrm{mag}$ brighter interior to a radial distance from the image centre of 50 arcsec. The outermost measurement however, deviates from the photographic profile by a negligible amount $(<0.01 \mathrm{mag})$.

CCD surface photometry has already been published by Vigroux et al. (1986) for IC 3475. Vigroux et al.'s profile of IC 3475 was directly comparable with our own smoothed elliptical surface-brightness profile. In both

\footnotetext{
7 These observations were not however amongst those used to calibrate the Schmidt plates.
}

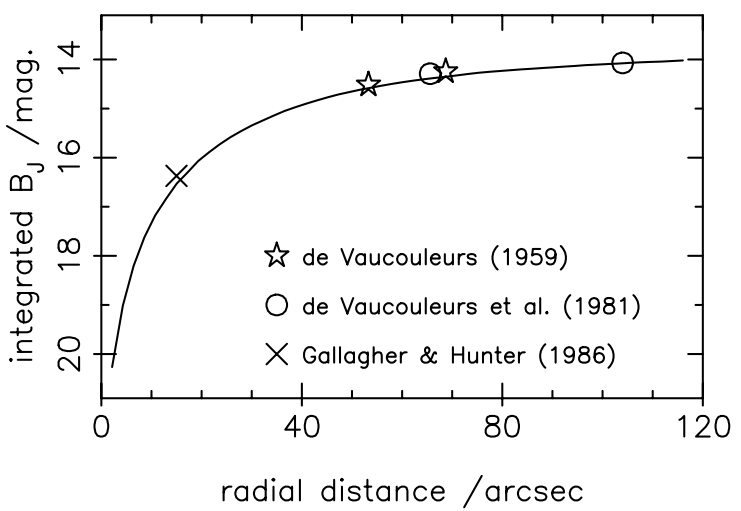

Fig. 19. Comparisons with previous $B$-band aperture-photometry measurements of IC 3475 , converted to $B_{\mathrm{J}}$ by means of Eq. (1), with $(B-V)=0.82$. All of the data points correspond to values tabulated previously. The curve represents the radially integrated profile from the unsmoothed image-scan of Plate J9229

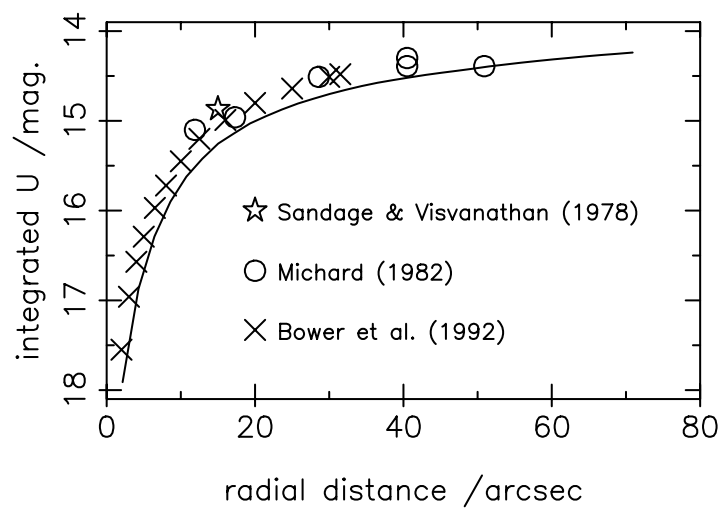

Fig. 20. Comparison with the $U$-band simulated-aperture CCD photometry of Bower et al. (1992) and the aperture photometry of Michard (1982) and Sandage \& Visvanathan (1978) for NGC 4468. The curve represents the radially integrated profile from the unsmoothed image-scan of Plate U9362

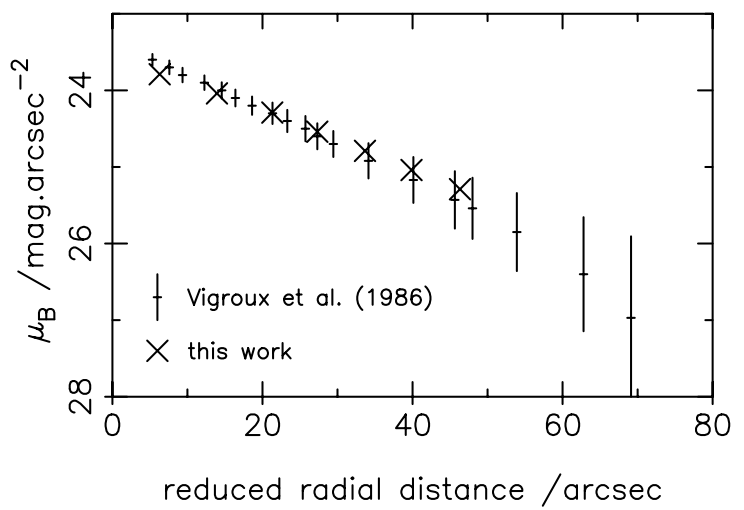

Fig. 21. Comparison between the CCD surface photometry of Vigroux et al. (1986) for IC 3475 and the smoothed photographic surface photometry on which the VPC is based. Vigroux et al.'s error bars are depicted 
cases, elliptical isophotes were fitted to the images and specified by the same reduced-radius parameter as well as by a position angle and an ellipticity. Conversion of $\mu_{B_{\mathrm{J}}}$ to $\mu_{B}$ was by means of Eq. (1) with a $(B-V)$ colour of 0.82. As can be seen from Fig. 21, there is a difference in profile slope, but the agreement between the photographic and CCD photometry is generally good, and well within Vigroux et al.'s quoted errors. The difference in profile slope is almost certainly due to the heavy smoothing applied to the VPC plate-scan data, as extrapolation of the two profiles to $r=\infty$ yields $B_{\mathrm{t}}=14.47$ for Vigroux et al.'s profile (using $n=1$ ) and $B_{\mathrm{t}}=14.5$ for the VPC profile (for which $n=1.2$ ).

Additional comparisons with CCD surface photometry were only possible in the cases of a few saturated galaxy images. This was because the published CCD surface photometry is confined to the very brightest galaxies in the Virgo Cluster, most of which were resolved into multiple images by the image-segmentation software. Those saturated galaxy images that did not suffer from image fragmentation, and for which relevant surface photometry could be found in the literature, are compared with these other sources in Fig. 22. Note that the VPC surface-brightness profiles plotted are based on the heavily smoothed plate-scan data.

As $(B-V)$ colour-gradient information was not available for NGC 4458 or NGC 4468, and Peletier et al. (1990) found no evidence for any colour gradient in the case of NGC 4551, mean $(B-V)$ colours from the RC3 were used for the transformations from the $B_{\mathrm{J}}$ system to the $B$ system. These colours were 0.90 for NGC 4458, 0.86 for NGC 4468 and 0.96 for NGC 4551. Note that Peletier et al.'s $R_{\mathrm{C}}$-band profile of NGC 4551 is likely to be more reliable than Jedrejewski's (1987) even though published aperture photometry was used for calibration purposes in both cases, as the former profile was the weighted mean of four different observations. It should also be noted that the exceptionally good agreement between Peletier et al.'s $R_{\mathrm{C}}$-band profile of NGC 4551 and the corresponding photographic profile, cannot be attributed wholly or even largely to the coincidence that the VPC's $R_{\mathrm{C}}$-band zero point was partially based on one of Peletier et al.'s four $R_{\mathrm{C}}$-band frames of that galaxy. This is because, not only did we reduce the [one] common CCD frame independently from Peletier et al., but our absolute calibration was based on on observations of standard stars (as described in Sect. 3.4) rather than on existing aperture-photometry measurements.

The apparent mean zero-point offsets for the $B$ profiles were $B$ (Boroson \& Thompson) $-B$ (VPC) $=+0.14$ $\left(\sigma_{N-1}=0.05\right) \mathrm{mag}, B$ (Peletier et al. $)-B(\mathrm{VPC})=-0.16$ $\left(\sigma_{N-1}=0.1\right) \mathrm{mag}, B($ Jedrejewski $)-B(\mathrm{VPC})=-0.19$ $\left(\sigma_{N-1}=0.1\right)$ mag. These mean residuals were computed for those parts of the profiles for which the reduced radius exceeded 20 arcsec, and $N$ in these cases refers to the number of data points on the relevant pro- file(s) compared (rather than the number of galaxies compared). Discrepancies between the photographic profiles and Peletier et al.'s $U$ and $R_{\mathrm{C}}$ profiles were negligible, though Jedrejewski's $R_{\mathrm{C}}$ profile was significantly brighter than both the photographic profile and Peletier et al.'s profile.

\subsection{Discussion}

From the comparisons made in this section, any major error in the zero points of the VPC's magnitude scales would seem unlikely, though it would appear possible that the true zero points could be slightly brighter than those adopted by as much as $\sim 0.1 \mathrm{mag}$, particularly in the case of the $B_{\mathrm{J}}$-band. One possibility is that Eq. (1) may not be as sound as it had been hoped and that its degree of applicability may vary significantly with galaxy type. In judging the $B_{\mathrm{J}}$-band comparisons made in this section, it must be remembered that the galaxy samples of other observers do not tend to cover all morphological types, but are often confined to a single type of object. Minor systematic discrepancies between the different mean residuals computed for different observers' photometry can therefore be expected.

There is still some disagreement as to the optimum relationship describing transformations between the UKST $B_{\mathrm{J}}$ system (IIIa-J, GG395) and the Johnson $B$ and $V$ systems. Equation (1) is identical to that used by Buta \& Corwin (1986). Blair \& Gilmore (1982) derived a colour term of $-0.28 \pm 0.04$ empirically, by plotting the colours of a large stellar sample, whilst Warren et al. (1991) derived a colour term of -0.35 by a similar method. The latter authors noted also that their empirical value disagreed with their predicted value of -0.26 , which was based on models of the manufacturer's filter transmission data and emulsion sensitivity functions. More recently, Metcalfe et al. (1995) have demonstrated the case for the colour term being at least as negative as -0.35 based on a galaxy sample.

It should also be remembered that even the unsmoothed plate-scan data suffered from relatively coarse sampling (2". 1 pixels) as well as relatively poor seeing. When other observers' photoelectric observations were made under good seeing conditions, slightly negative mag(other work) - mag(VPC) residuals would be expected for small apertures. Although this bias would make the VPC zero points look slightly fainter in these comparisons, it is not applicable to the VPC's $B_{\mathrm{J}}$-total magnitude scale, nor does it have more than second-order consequences for the VPC's isophotal magnitudes (as is evident from Table 8).

In conclusion, the exact zero-point errors in the VPC's magnitude scales cannot be estimated with confidence on the basis of the existing photoelectric measurements for Virgo galaxies in the literature, which exhibit some mutual disagreements. However, on the basis of all of the 

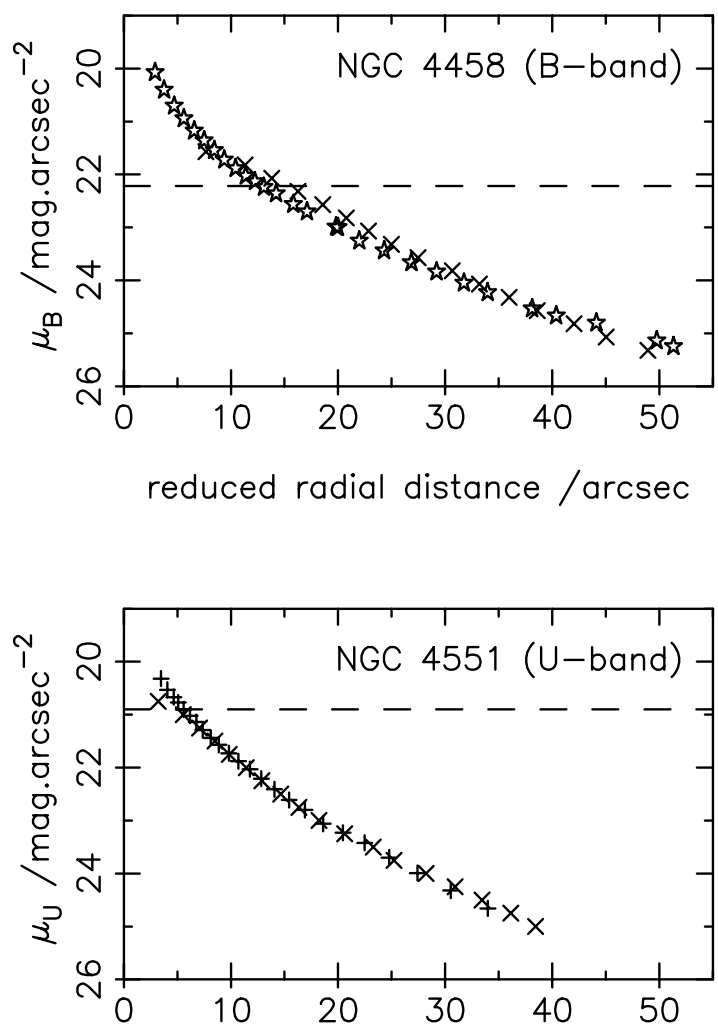

reduced radial distance / arcsec

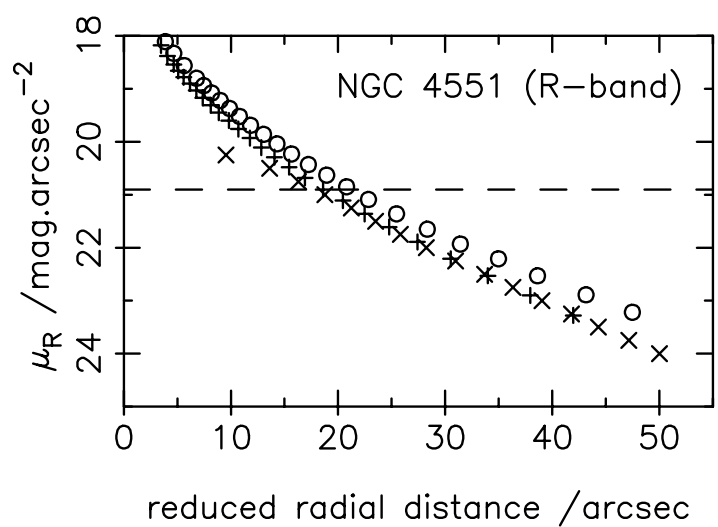

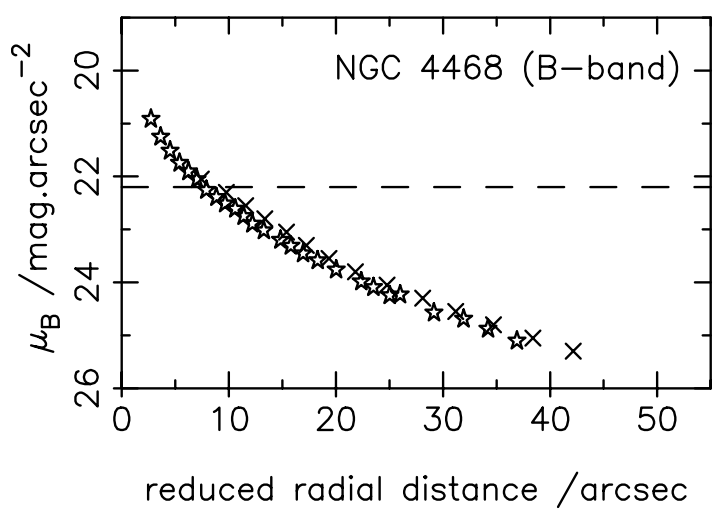

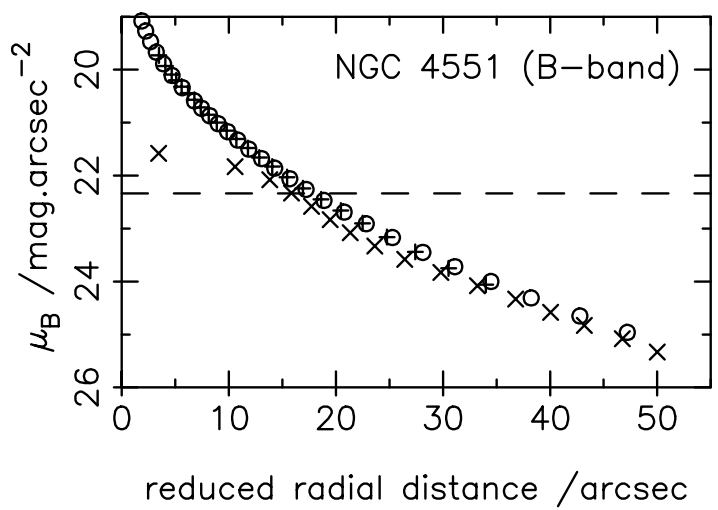

is Boroson \& Thompson (1987)
O Jedrejewski (1987)
+ Peletier et al. (1990)
$\times$ this work

Fig. 22. Comparisons of saturated [and smoothed] photographic profiles generated during the compilation of the VPC with other observers' CCD surface-photometry. In most cases, the extent of the saturated core within the photographic image is clearly discernable. The dashed lines represent the bright isophote limits listed in Table 5

comparisons made in this section, our best estimates of the observed $U$-band and $B_{\mathrm{J}}$-band zero-point discrepancies are -0.10 and -0.15 magnitudes respectively. It cannot be overemphasized that a simple weighted mean of the zero-point discrepancies found with respect to existing photoelectric aperture and CCD photometry (which would give most weight to Caldwell's 1983 measurements) would be unlikely to yield a meaningful result because most observers did not document the FWHM of their seeing discs and because of the variation in the aperture sizes used (with Caldwell's apertures being amongst the small- est). It is probably fair to assume that most observers' photometry had the twin advantages of better seeing and sampling than the VPC's (even before smoothing) as discussed in the previous paragraph. The actual zero-point errors are therefore almost without any doubt smaller than the observed discrepancies. The overall very provisional zero-point error estimates are therefore mag(other work $)-\operatorname{mag}(\mathrm{VPC})=0.0_{-0.05}^{+0.0}$ and $0.0_{-0.1}^{+0.0}$ for the $U$ and $B \mathrm{~J}$ bands respectively. In the case of the $R_{\mathrm{C}}$-band magnitude scale, there is very little photometry in the literature, and meaningful comparisons with other sources were not 
possible until Durrell's (1997) observations of several dwarfs that are also [unsaturated] VPC objects. Durrell (1997) has already noted the excellent agreement he found between his total magnitudes (which were derived from CCD observations) and those of Young \& Currie (1995), hereunder YC3, which he based on three dwarf galaxies in common between the two samples ( $T 1$ (Durrell) $\left.R_{\mathrm{C}}(\mathrm{YC} 3)=-0.04 \pm 0.07 \mathrm{mag}\right)$. As described in Sects. 4.2 and 4.3 , we have improved our total-magnitude extrapolation procedure since $\mathrm{YC} 3$. We now find an even smaller zero-point offset of $T 1$ (Durrell) $-R_{\mathrm{C}}(\mathrm{VPC})=-0.03 \pm$ 0.06 mag based on the same three galaxies.

Acknowledgements. We thank the UKST Unit for loan of the plate material; the ROE's Image \& Data Processing Unit for scanning the plates with COSMOS; Starlink, Oxford University Computing Service, the QSO \& Observational Cosmology Group at BAO and Wu Xiang-ping for use of their computing facilities; Tony Lynas-Gray for maintaining the Oxford astrophysics VAX cluster beyond its period of support by Starlink; Jon Godwin for his software environment and in particular for his image-segmentation software; Reynier Peletier for several CCD frames; Michael Drinkwater for spotting a galaxy that we had overlooked and Bruno Binggeli for a machine-readable version of the VCC. CKY gratefully acknowledges a SERC studentship for the early part of this work and a PDRF from the National Postdoctoral Fellowship Office of China for the later part.

\section{References}

Binggeli B., Popescu C.C., Tammann G.A., 1993, A\&AS 98, 275

Binggeli B., Sandage A., Tammann G.A., 1985, AJ 90, 1681 (VCC)

Binggeli B., Sandage A., Tarenghi M., 1984, AJ 89, 64

Binggeli B., Tammann G.A., Sandage A., 1987, AJ 94, 251

Blair M., Gilmore G., 1982, PASP 94, 742

Boroson T.A., Thompson I.B., 1987, AJ 92, 33

Bothun G.D., Mould J.R., Caldwell N., MacGillivray H.T., 1986, AJ 92, 1007

Bower R.G., Lucey J.R., Ellis R.S., 1992, MNRAS 254, 589

Burstein D., Davies R.L., Dressler A., et al., 1987, ApJS 64, 601

Buta R.J., Corwin H.G., 1986, ApJS 62, 255

Caldwell N., 1983, AJ 88, 804

Carter D., Godwin J.G., 1979, MNRAS 187, 711

de Vaucouleurs A., Longo G., 1985, Monographs in Astronomy No. 5, University of Texas, Austin

de Vaucouleurs G., 1959, Lowell Obs. Bull. 4, 97

de Vaucouleurs G., Corwin H.G., 1986, AJ 92, 722

de Vaucouleurs G., Pence W.D., 1979, ApJS 40, 425

de Vaucouleurs G., de Vaucouleurs A., Buta R., 1981, AJ 86, 1429

de Vaucouleurs G., de Vaucouleurs A., Corwin H.G., 1976, Second Reference Catalogue of Bright Galaxies, University of Texas, Austin de Vaucouleurs G., de Vaucouleurs A., Corwin H.G., 1978, AJ 83,1331

de Vaucouleurs G., de Vaucouleurs A., Corwin H.G., Buta R.J., Paturel G., Fouque P., 1991, Third Reference Catalogue of Bright Galaxies. Springer-Verlag, New York (RC3)

Dixon K.L., 1979, D. Phil. thesis, University of Oxford

Drinkwater M.J., Currie M.J., Young C.K., Hardy E., Yearsley J.M., 1996, MNRAS 279, 595

Drinkwater M., Hardy E., 1991, AJ 101, 94

Durrell P.R., 1997, AJ 113, 531

Fukugita M., Okamura S., Yasuda N., 1993, ApJ 412, L13

Gallagher J.S., Hunter D.A., 1986, AJ 92, 557

Godwin J.G., 1976, D. Phil. thesis, University of Oxford

Heckmann O., Diekvoss W., Kox H., Gunther A., Brosterhus E., 1975, AGK3; Star Catalogue of Positions and Proper Motions, Hamburger Sternwarte, Hamburg

Jacoby G.H., Branch D., Ciardullo R., et al., 1992, PASP 104, 599

Jedrejewski R.I., 1987, MNRAS 226, 747

Karachentsev I.D., Karachentseva V.E., 1982, Pis'ma Astron. Zh. 8, 198

Karachentsev I.D., Karachentseva V.E., Borngen F., 1983, Pis'ma Astron. Zh. 9, 387

Landolt A.U., 1983, AJ 88, 439

Longo G., de Vaucouleurs A., 1983, Monographs in Astronomy No. 3, University of Texas, Austin

Longo G., de Vaucouleurs A., 1985, Monographs in Astronomy No. 3A, University of Texas, Austin

MacGillivray H.T., Martin R., Pratt N.M., et al., 1976, MNRAS 186, 69

Metcalfe N., Fong R., Shanks T., 1995, MNRAS 274, 769

Michard R., 1982, A\&AS 49, 591

Newell B., O'Neil E.J., 1977, PASP 89, 925

Oemler A., 1974, ApJ 194, 1

Peletier R.F., Davies R.L., Illingworth G.D., Davis L.E., Cawson M., 1990, AJ 100, 1091

Phillipps S., Davies J.I., Disney M.J., 1988, MNRAS 233, 485

Pierce M.J., Tully R.B., 1988, ApJ 330, 579

Poulain P., 1988, A\&AS 72, 215

Rhee G.F.R.N., Katgert P., 1988, A\&AS 72, 243

Sandage A., Visvanathan N., 1978, ApJ 223, 707

Sérsic J.L., 1968, Atlas de galaxias australes. Observatorio Astronomica, Cordoba

Tonry J.L., 1991, ApJ 373, L1

Tonry J.L., Ajhar E.A., Luppino G.A., 1990, AJ 100, 1416

Vigroux L., Thuan T.X., Vader J.P., Lachieze-Rey M., 1986, AJ 91, 70

Warren S.J., Hewett P.C., Irwin M.J., Osmer P.S., 1991, ApJS 76,1

Young C.K., 1994, D. Phil. thesis, University of Oxford

Young C.K., Galaxies. In: Chan K.C., Cheng K.S. (eds.) Proceedings of 21st Century Chinese Astronomy Conference. World Scientific Publishing, Singapore

Young C.K., Currie M.J., 1991, Observatory 111, 220

Young C.K., Currie M.J., 1994, MNRAS 268, L11

Young C.K., Currie M.J., 1995, MNRAS 273, 1141

Young C.K., Metcalfe N., Zhu J., Wu H., Chen J., A\&A (in press) 
Appendix A:
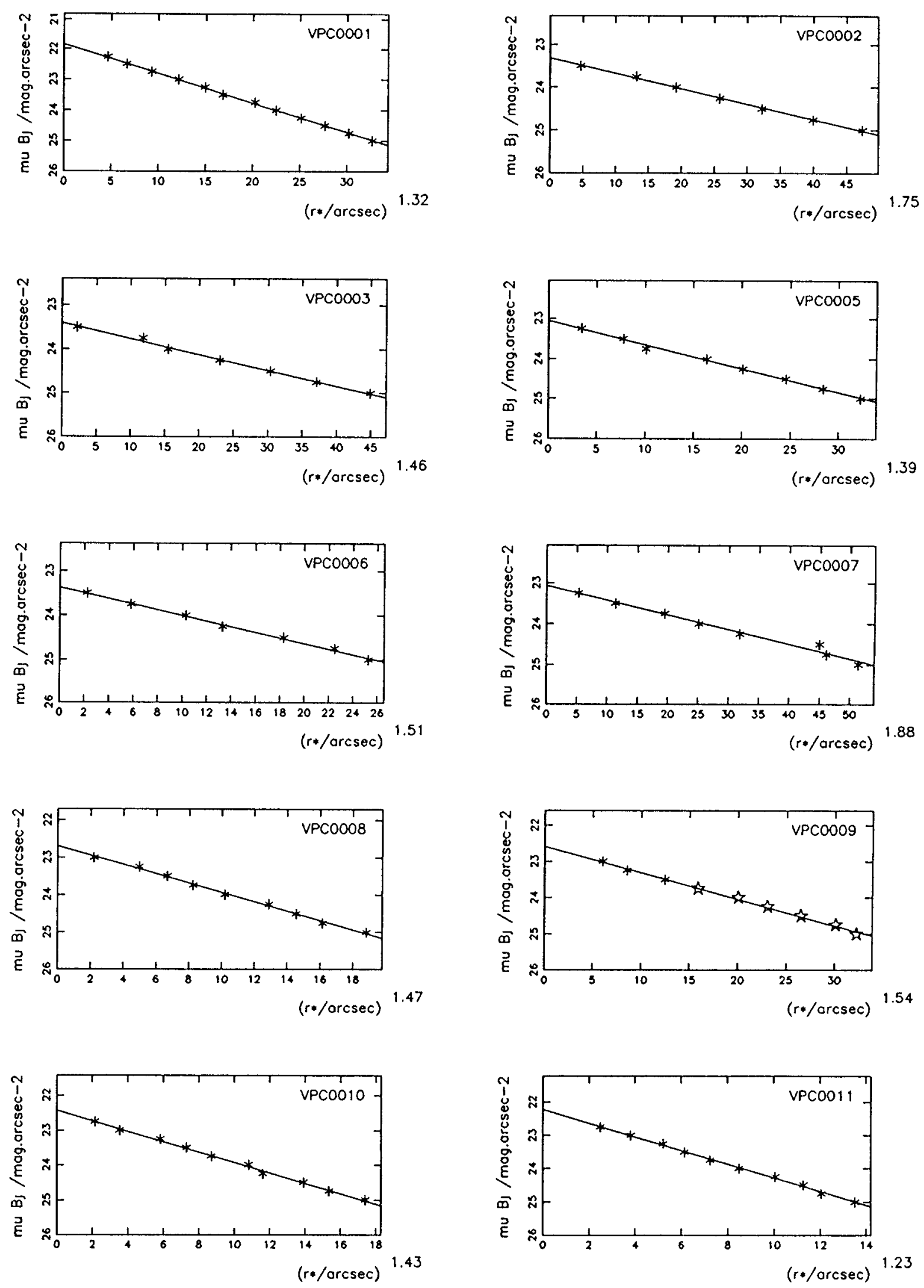

Fig. 1. Example fits to galaxy surface-brightness profiles ( $\mathrm{r}^{*}$ denotes reduced radius) 

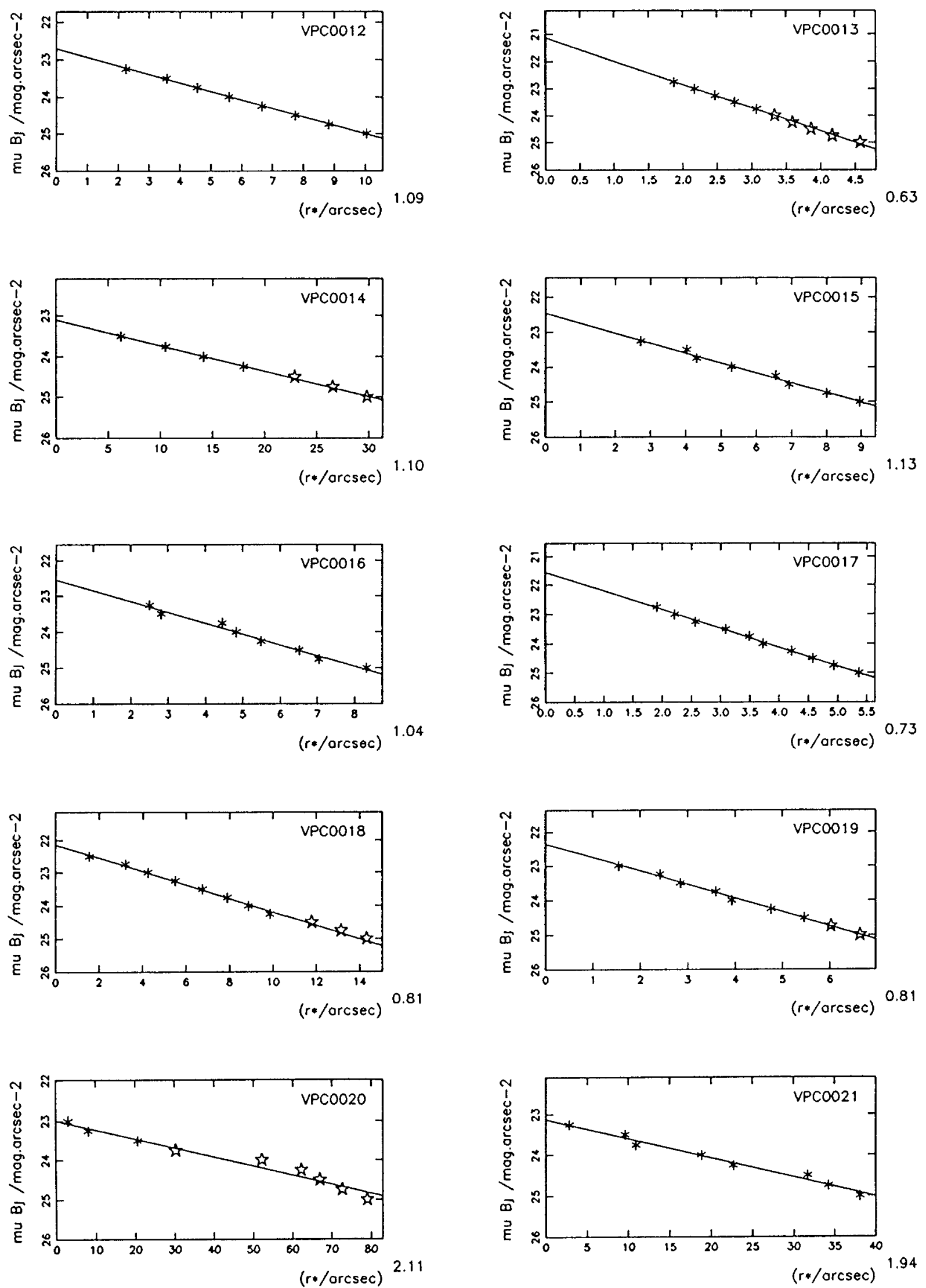

Fig. 1. continued 

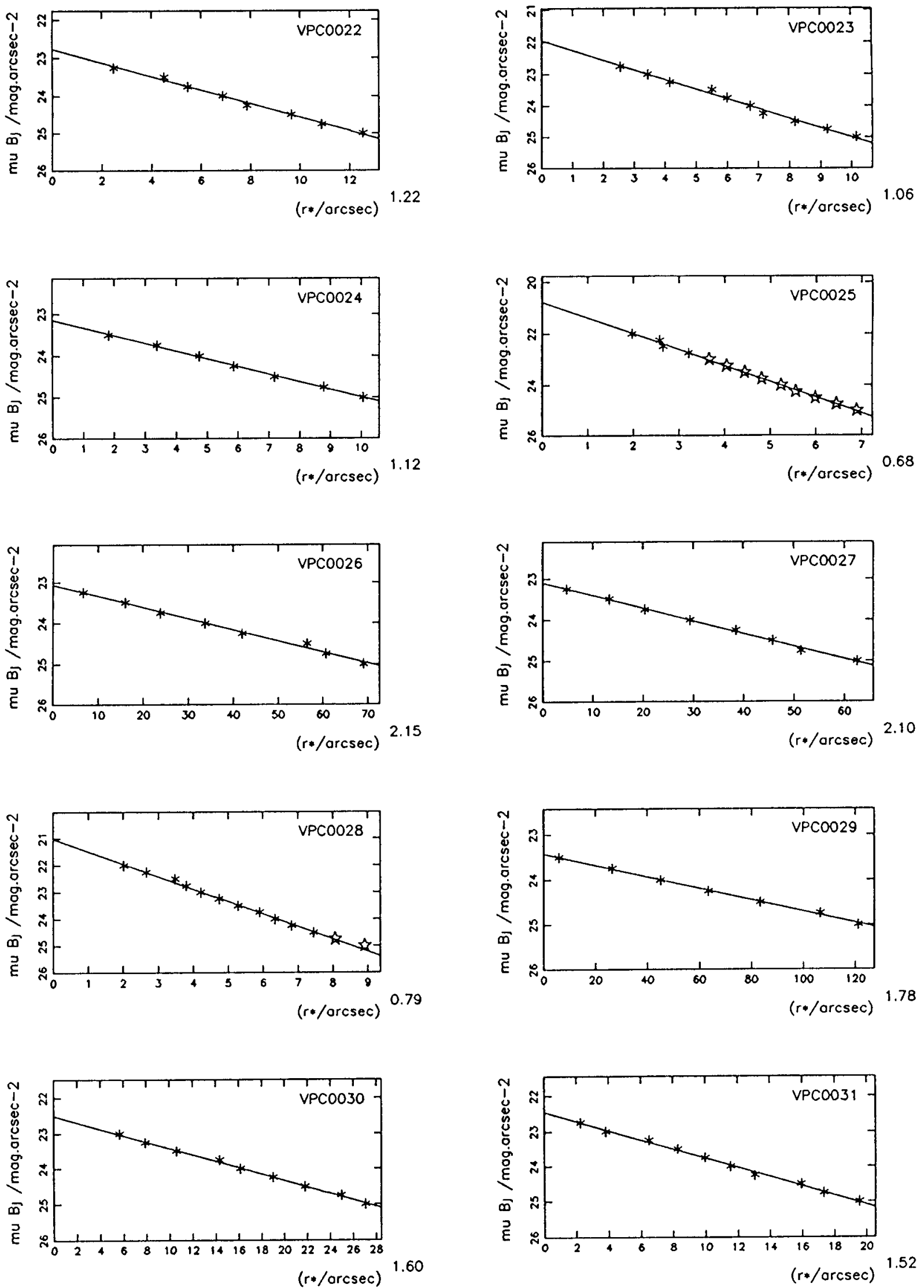

Fig. 1. continued 
Appendix D: The extension to the main catalogue.

Table 14. Inextricably merged objects, which are designated VPCX to distinguish them from main-catalogue objects

\begin{tabular}{|c|c|c|c|c|c|}
\hline VPCX & $(\alpha-12 \mathrm{~h}, \delta)_{1950.0}$ & $n(\text { gal.s })^{\mathrm{a}}$ & $B_{\mathrm{J}_{25}}^{\mathrm{b}}$ & $\sigma_{B_{\mathrm{J}_{25}}}$ & notes \\
\hline $1 \mathrm{~A}, \mathrm{~B}$ & $1623.4+124322$ & 2 & 17.1 & 0.2 & interacting pair \\
\hline 2 & $1707.1+124010$ & 1 & 17.5 & 0.3 & star subtracted \\
\hline $3 \mathrm{~A}, \mathrm{~B}$ & $1711.2+134342$ & 2 & 17.3 & 0.2 & including small companion at end of spiral arm \\
\hline $4 \mathrm{~A}, \mathrm{~B}$ & $1809.3+114042$ & 2 & 17.6 & 0.2 & interacting pair \\
\hline 5 & $1832.6+120541$ & 1 & 17.0 & 0.5 & star subtracted \\
\hline 6 & $1923.5+130724$ & 1 & 17.7 & 0.5 & star subtracted \\
\hline 7 & $2008.6+115128$ & 1 & 18.6 & 0.5 & star subtracted \\
\hline $8 \mathrm{~A}, \mathrm{~B}$ & $2023.9+123409$ & 2 & 17.8 & 0.2 & interacting pair \\
\hline $9 \mathrm{~A}, \mathrm{~B}$ & $2039.7+131811$ & 2 & 17.4 & 0.4 & $\begin{array}{l}\text { a third fainter galaxy subtracted, } \\
\text { leaving two interacting ones }\end{array}$ \\
\hline 10 & $2115.8+130004$ & 1 & 18.2 & 0.5 & star subtracted \\
\hline 11 & $2128.6+105715$ & 1 & 18.6 & 0.4 & one fainter galaxy subtracted \\
\hline 12 & $2149.9+131814$ & $1 \mathrm{E} ?$ & 15.7 & 1.0 & bright star subtracted during segmentation \\
\hline 13 & $2152.7+152110$ & 1 & 17.3 & 0.5 & star subtracted \\
\hline $14 \mathrm{~A}, \mathrm{~B}$ & $2156.2+133941$ & 2 & 17.4 & 0.2 & probably connected \\
\hline 15 & $2227.4+121614$ & 1 & 18.3 & 1.0 & $\begin{array}{l}\text { most probably star and galaxy; } \\
\text { star suspect subtracted }\end{array}$ \\
\hline $16 \mathrm{~A}$ & $2243.4+130914$ & 1 & 18.5 & 0.8 & background subtraction problems \\
\hline $16 \mathrm{~B}$ & $" n$ & 1 & 18.5 & 0.8 & background subtraction problems \\
\hline 17 & $2256.7+134627$ & 1 & 18.4 & 0.5 & star subtracted \\
\hline 18 & $2412.2+111142$ & $1 \mathrm{~S}$ & 16.6 & 0.5 & star subtracted \\
\hline 19 & $2412.4+113056$ & 1 & 18.1 & 0.5 & one fainter galaxy subtracted \\
\hline 20 & $2446.0+142523$ & 1 & ? & . & possible galaxy behind star \\
\hline $21 \mathrm{~A}, \mathrm{~B}$ & $2525.3+110912$ & 2 & 18.1 & 0.2 & interacting pair \\
\hline $22 \mathrm{~A}, \mathrm{~B}$ & $2624.6+104735$ & 2 & 17.9 & 0.2 & interacting pair \\
\hline 23 & $2654.6+143111$ & 1 & 17.7 & 0.5 & star subtracted \\
\hline $24 \mathrm{~A}, \mathrm{~B}$ & $2708.4+123417$ & 2 & 17.8 & 0.2 & interacting pair \\
\hline 25 & $2710.5+114037$ & $1 \mathrm{~S} 0 ?$ & 16.0 & 0.5 & star subtracted \\
\hline 26 & $2718.4+120204$ & 1 & 18.6 & 0.5 & star subtracted \\
\hline $27 \mathrm{~A}, \mathrm{~B}$ & $2720.3+115714$ & $2 \mathrm{E}$ & 15.7 & 0.2 & interacting pair \\
\hline 28 & $2739.6+120113$ & $1 \mathrm{E}$ & 16.6 & 0.5 & poorly resolved during segmentation \\
\hline 29 & $2740.0+120115$ & $1 \mathrm{E}$ & 16.5 & 0.5 & poorly resolved during segmentation \\
\hline 30 & $2750.8+110844$ & 1 & 17.5 & 0.5 & star subtracted \\
\hline 31 & $2752.6+112758$ & 1 & 17.8 & 0.4 & one fainter galaxy subtracted \\
\hline 32 & $2802.8+130152$ & 1 & 17.7 & 0.5 & star subtracted \\
\hline $33 \mathrm{~A}, \mathrm{~B}$ & $2811.0+105117$ & 2 & 18.2 & 0.2 & interacting pair \\
\hline 34 & $2834.0+105322$ & 1 & 18.6 & 0.3 & star subtracted \\
\hline 35 & $2845.9+140616$ & $1 \mathrm{~S} ?$ & 16.8 & 0.5 & star subtracted \\
\hline $36 \mathrm{~A}, \mathrm{~B}$ & $2848.9+151330$ & 2 & 17.8 & 0.2 & probably interacting \\
\hline 37 & $2854.8+131131$ & 1 & 17.8 & 0.5 & star subtracted \\
\hline $38 \mathrm{~A}, \mathrm{~B}$ & $2909.9+152425$ & 2 & 18.6 & 0.2 & interacting pair \\
\hline 39 & $2920.6+150506$ & 1 & 18.3 & 0.5 & star subtracted \\
\hline 40 & $2923.4+133247$ & 1 & 18.4 & 0.5 & star subtracted \\
\hline $41 \mathrm{~A}, \mathrm{~B}$ & $3014.2+152034$ & 2 & 18.6 & 0.2 & interacting pair \\
\hline 42 & $3029.3+112541$ & $1 \mathrm{~S} ?$ & 16.8 & 0.3 & star subtracted \\
\hline $43 \mathrm{~A}, \mathrm{~B}$ & $3138.7+152556$ & 2 & 17.7 & 0.2 & interacting pair \\
\hline 44 & $3151.7+122023$ & 1 & 17.9 & 0.3 & star subtracted \\
\hline 45 & $3223.6+152635$ & 1 & 17.6 & 0.5 & star subtracted \\
\hline $46 \mathrm{~A}, \mathrm{~B}$ & $3303.6+112501$ & 2 & 18.1 & 0.2 & interacting pair \\
\hline $47 \mathrm{~A}, \mathrm{~B}$ & $3325.9+113250$ & $2 \mathrm{E}$ & 16.7 & 0.2 & interacting pair \\
\hline 48 & $3343.1+145638$ & 1 & 18.8 & 0.5 & star subtracted \\
\hline $49 \mathrm{~A}, \mathrm{~B}$ & $3352.5+131202$ & 2 & 17.4 & 0.2 & interacting pair \\
\hline 50 & $3407.8+112237$ & 1S0 & 15.9 & 0.3 & IC 3578 ; star subtracted \\
\hline 51 & $3438.7+145933$ & 1 & 17.5 & 0.5 & star subtracted \\
\hline
\end{tabular}

\footnotetext{
${ }^{\text {a }} n$ (gal.s) $=$ number of galaxies observed for which $B_{\mathrm{J}_{25}} \leq 19.0$; morphological types quoted if $B_{\mathrm{J}_{25}} \leq 17.0$

${ }^{\mathrm{b}}$ combined light due to all galaxies for which $B_{\mathrm{J}_{25}} \leq 19.0$.
} 
Appendix F: NGC and IC designations for VPC objects.

Table 16. VPC objects with IC designations

Table 15. VPC objects with NGC designations

\begin{tabular}{rrrr}
\hline VPC & NGC & VPC & NGC \\
\hline 49 & 4262 & 622 & 4458 \\
63 & 4267 & 626 & 4459 \\
130 & 4294 & 629 & 4461 \\
146 & 4298 & 665 & 4468 \\
149 & 4299 & 687 & 4473 \\
155 & 4302 & 700 & 4474 \\
178 & 4305 & 706 & 4476 \\
179 & 4306 & 711 & 4477 \\
222 & 4313 & 722 & 4482 \\
242 & 4320 & 736 & 4478 \\
250 & 4325 & 741 & 4479 \\
271 & 4330 & 755 & $4486 \mathrm{~B}$ \\
324 & 4351 & 771 & 4486 \\
328 & 4352 & 778 & 4491 \\
375 & 4371 & 780 & $4486 \mathrm{~A}$ \\
385 & 4374 & 820 & 4497 \\
394 & 4377 & 840 & 4501 \\
432 & 4387 & 849 & 4503 \\
438 & 4388 & 855 & 4506 \\
441 & 4390 & 894 & 4516 \\
452 & 4402 & 919 & 4523 \\
454 & 4406 & 935 & 4528 \\
472 & 4413 & 948 & 4531 \\
511 & 4419 & 995 & 4548 \\
518 & 4425 & 1002 & 4550 \\
531 & 4429 & 1010 & 4551 \\
532 & 4431 & 1012 & 4552 \\
542 & 4435 & 1050 & 4564 \\
544 & 4436 & 1058 & 4567 \\
550 & 4438 & 1064 & 4568 \\
560 & 4440 & 1082 & 4569 \\
574 & 4446 & 1088 & 4571 \\
582 & 4447 & 1119 & 4579 \\
613 & 4452 & & \\
\hline & & &
\end{tabular}

\begin{tabular}{rrrr}
\hline VPC & IC & VPC & IC \\
\hline 4 & 775 & 704 & 3425 \\
18 & 3142 & 751 & 3432 \\
36 & 3147 & 760 & 3435 \\
40 & 3149 & 765 & 3437 \\
78 & 781 & 801 & 3443 \\
99 & 3173 & 810 & 3442 \\
114 & 3187 & 813 & 3446 \\
116 & 3188 & 827 & 3453 \\
159 & 3199 & 834 & 3457 \\
182 & 3209 & 838 & 797 \\
252 & 3238 & 839 & 3459 \\
256 & 3239 & 843 & 3461 \\
260 & 3244 & 847 & 3466 \\
301 & 3258 & 864 & 3467 \\
356 & 3284 & 865 & 3470 \\
395 & 3305 & 878 & 3475 \\
397 & 3303 & 881 & 3476 \\
419 & 3311 & 884 & 3478 \\
446 & 3327 & 885 & 3481 \\
447 & 3331 & 887 & $3481 \mathrm{~A}$ \\
474 & 3344 & 897 & 3483 \\
496 & 3349 & 898 & 3490 \\
502 & 3355 & 900 & 3489 \\
505 & 3356 & 901 & 3492 \\
507 & 3358 & 918 & 3499 \\
514 & 3363 & 920 & 3500 \\
528 & 3371 & 923 & 350 \\
575 & 794 & 943 & 3509 \\
589 & 3381 & 947 & 3510 \\
600 & 3388 & 960 & 3520 \\
610 & 3393 & 973 & 3522 \\
615 & 3392 & 996 & 3540 \\
651 & 3413 & 1047 & 3567 \\
670 & 3416 & 1073 & 3583 \\
682 & 3418 & 1085 & 3586 \\
\hline & & &
\end{tabular}

